\title{
Impact of violations of measurement invariance in cross-lagged panel mediation models
}

\author{
Jie Xu ${ }^{1}$ (1) - Qian Zhang ${ }^{1} \cdot$ Yanyun Yang ${ }^{1}$ \\ Published online: 9 June 2020 \\ (C) The Psychonomic Society, Inc. 2020
}

\begin{abstract}
When fitting cross-lagged panel mediation models, the assumption of longitudinal measurement invariance should be made. This simulation study investigated the impact of violations of measurement invariance on cross-lagged panel mediation analysis using the latent cross-lagged panel mediation model (L-CLPM). Results showed that estimates of direct and mediated effects tended to be less accurate and the type I error rate of testing mediated effects can be inflated under non-invariance conditions. On the other hand, power for detecting model misspecifications due to longitudinal non-invariance was high across all investigated conditions. Fit indices were not sensitive to violations of measurement invariance in the L-CLPM under the manipulated conditions. We end the article with suggestions, limitations, and future directions.
\end{abstract}

Keywords Measurement invariance $\cdot$ Cross-lagged panel mediation $\cdot$ Model fit $\cdot$ Parameter estimation

Mediation modeling has been widely used in behavioral and social sciences. Most applications of mediation models are based on cross-sectional designs where data are collected on the same occasion. However, researchers have argued against the practice of cross-sectional meditation analysis (e.g., Cole \& Maxwell, 2003; Gollob \& Reichardt, 1987; Maxwell \& Cole, 2007; Maxwell, Cole, \& Mitchell, 2011). Conceptually, implied causal relationships in the mediation process take time to unfold and therefore cross-sectional designs are not appropriate. Statistically, Maxwell and colleagues $(2007,2011)$ found that cross-sectional mediation models produce inaccurate estimates of mediation effects when the underlying mediation process is longitudinal.

To reveal longitudinal mediated effects, longitudinal designs have been strongly recommended. Different longitudinal mediation models have been proposed for mediation analysis, such as the cross-lagged panel mediation (CLPM; Cole

Jie Xu

jx12@my.fsu.edu

Qian Zhang

qzhang4@fsu.edu

Yanyun Yang

yyang3@admin.fsu.edu

1 Measurement \& Statistics, Florida State University, Tallahassee, FL 32306-4453, USA
\& Maxwell, 2003), the latent growth mediation (LGM, Cheong, MacKinnon, \& Khoo, 2003; MacKinnon, 2008; Soest \& Hagtvet, 2011), and the latent change score mediation (LCSM; MacKinnon, 2008; Selig \& Preacher, 2009) models. Selection of an appropriate longitudinal mediation model can be made based on research questions of interest. When causal mediation effects among variables measured over time are of interest, CLPM models are more appropriate (MacKinnon, 2008; Selig \& Preacher, 2009). LGM models focus on the mediation process in initial status and latent growth rate among variables. For LCSM models, change scores between two time points are used for mediation analysis. Among these three types of longitudinal mediation models, we focus on CLPM models that address questions of how a predictor $X$ influences an outcome $Y$ via a mediator $M$ over time, while accounting for effects of previous measures of the same variables. Applications of CLPM models can be found in different fields of research (e.g., Jaya, Ascone, \& Lincoln, 2018; Pardini, Loeber, \& Stouthamer-Loeber, 2005; Watts, Webb, \& Netuveli, 2017).

A CLPM model may be applied in two forms. In the first form, $X, M$, and $Y$ is each represented by an observed variable. In the cases when $X, M$, and $Y$ are measured by multiple items, a composite score (e.g., mean score) across items is used to represent $X, M$, and $Y$. We label this applied model as the observed cross-lagged panel mediation model (O-CLPM). The O-CLPM has been used in assessing the mediated effect in applied studies with longitudinal data (e.g., Watts, Webb, \& 
Netuveli, 2017), but limitations exist in applying O-CLPM. First, measurement errors cannot be accounted for. Failure to account for measurement errors in observed scores can result in biased estimates of direct and indirect effects in mediation analysis (Bentley, 2011; Cole \& Maxwell, 2003; Judd \& Kenny, 1981; Zhang \& Yang, in press). Second, longitudinal measurement invariance, which is an important assumption in longitudinal modeling, cannot be tested (Selig \& Little, 2012).

Considering limitations of the O-CLPM, Cole and Maxwell (2003) proposed and recommended the second form of CLPM, the latent cross-lagged panel mediation model (LCLPM). The L-CLPM uses latent $X, M, Y$ measured by multiple indicators to account for measurement errors and enable evaluating longitudinal measurement invariance. The LCLPM is the model of interest in the current study.

\section{The latent cross-lagged panel mediation model}

Let $X, M$, and $Y$ be the latent predictor, mediator, and outcome respectively, and $t$ be a specific occasion $(t=1,2, \ldots, T)$. An L-CLPM with lag-1 effects ${ }^{1}(X, M$, and $Y$ at time $t$ are predicted by those at time $t-1$ ) can be written as (see also Fig. 1):

$X_{t}=U_{t}^{(X)}+d_{\mathrm{x}} \cdot X_{t-1}+e_{t}^{(X)}$,

$M_{t}=U_{t}^{(M)}+d_{\mathrm{m}} \cdot M_{t-1}+a \cdot X_{t-1}+e_{t}^{(M)}$,

$Y_{t}=U_{t}^{(Y)}+d_{\mathrm{y}} \cdot Y_{t-1}+b \cdot M_{t-1}+c^{\prime} \cdot X_{t-1}+e_{t}^{(Y)}$,

where $d_{\mathrm{x}}, d_{\mathrm{m}}$, and $d_{\mathrm{y}}$ are the autoregressive path coefficients between two adjacent measurements of $X, M$, and $Y . U_{t}^{(X)}$, $U_{t}^{(M)}$, and $U_{t}^{(Y)}$ are the intercepts at time $t$ for the latent factors $X, M$, and $Y . e_{t}^{(X)}, e_{t}^{(M)}$, and $e_{t}^{(Y)}$ are the residuals for $X, M$, and $Y$; when $t=1$, residual variances are the variances of $X, M$, and $Y$. The parameters of interest include $a, b$, and $c^{\prime}$ ,which are the effects of $X_{t-1}$ on $M_{t}$ controlling for $M_{t-1}, M_{t-}$ ${ }_{1}$ on $Y_{t}$ controlling for $X_{t-1}$ and $Y_{t-1}$, and $X_{t-1}$ on $Y_{t}$ controlling for $M_{t-1}$ and $Y_{t-1}$ respectively. In addition, we are also interested in the specific mediated effect from $X_{t-2}$ to $Y_{t}$ via $M_{t-1}(a b)$.

Let $X, M$, and $Y$ be measured by $P, Q$, and $H$ items, respectively. The measurement components of the L-CLPM displayed in Fig. 1 can be expressed as:

\footnotetext{
$\overline{1}$ Another CLPM model discussed by Cole and Maxwell (2003) has direct effects from $X$ at time $t-2$ to $Y$ at time $t$. We considered the scenario of lag-1 direct effect in the L-CLPM for the following two reasons. First, it is not uncommon to observe significant lag-1 direct effects in applied studies using observed or latent cross-lagged panel mediation models (e.g., Hanson et al., 2016; Pardini, Loeber, \& Stouthamer-Loeber, 2005). Second, theoretically speaking, it is more likely to observe lag-1 or both lag-1 and lag-2 direct effects, than lag-2 direct effect only.
}

$X_{p t}=V_{p t}^{(X)}+\lambda_{p t}^{(X)} \cdot X_{t}+e_{p t}^{(X)}$,

$M_{q t}=V_{q t}^{(M)}+\lambda_{q t}^{(M)} \cdot M_{t}+e_{q t}^{(M)}$,

$Y_{h t}=V_{h t}^{(Y)}+\lambda_{h t}^{(Y)} \cdot Y_{t}+e_{h t}^{(Y)}$,

where $X_{p t}$ is the $p$ th item of the latent factor $X$ at time $t, M_{q t}$ is the $q$ th item of the latent factor $M$ at time $t$, and $Y_{h t}$ is the $h$ th item of the latent factor $Y$ at time $t . V_{p t}^{(X)}, V_{q t}^{(M)}$, and $V_{h t}^{(Y)}$ are the item intercepts. $\lambda_{p t}^{(X)}, \lambda_{q t}^{(M)}$, and $\lambda_{h t}^{(P)}$ are the loadings. $e_{p t}^{(X)}$, $e_{q t}^{(M)}$ and $e_{h t}^{(Y)}$ are the corresponding error terms.

Common to many other mediation models, assumptions such as the correct functional form and no omitted confounding variables are needed for CLPM models (Mackinnon, 2008). ${ }^{2}$ Mediation can be seen as a longitudinal process considering embedded causal sequences: a presumed predictor is measured prior to the presumed mediator, and the presumed mediator is measured prior to the presumed outcome (e.g., Cole \& Maxwell, 2003; MacKinnon, 2008). This assumption of temporal ordering is required to establish a causal mediated effect.

In addition to these assumptions, three important assumptions are made when fitting a CLPM model: stability, stationarity, and equilibrium (Kenny, 1979). Stability means that there is no growth over time for $X, M$, and $Y$. If this assumption holds, the relative standings (rank orders) of individuals do not change across time. Stationarity means that the relations of $X$ and $M, M$ and $Y$, and $X$ and $Y$ are invariant over time. Stationarity can be understood as "unchanging causal structure" across time (Kenny, 1979, p. 232). Equilibrium means variances and covariances among $X, M$, and $Y$ are the same across measurement occasions. The interpretation of identified mediated effects depends on the degree to which these assumptions hold in the CLPM model (Cole \& Maxwell 2003, Kenny, 1979; MacKinnon, 2008). If these assumptions are not met, it would be difficult to explain the mediated effects. Another less discussed yet important assumption for CLPM models is the longitudinal measurement invariance. This assumption cannot be tested in the O-CLPM but can be tested in the L-CLPM.

\section{Longitudinal measurement invariance}

Recently, attention has been called to the importance of establishing measurement invariance in longitudinal studies (e.g., Boom, 2015; Liu et al., 2016; Meredith, \& Horn, 2001; Millsap \& Cham, 2012; Widaman, Ferrer, \& Conger, 2010).

\footnotetext{
${ }^{2}$ Relying on counterfactuals and no unmeasured confounding assumptions, researchers have proposed a general framework to identify causal mediated effects independent of the statistical model (e.g., Valeri \& VanderWeele, 2013). In contrast, we focus on a latent variable modeling framework that allows examination of longitudinal measurement invariance.
} 


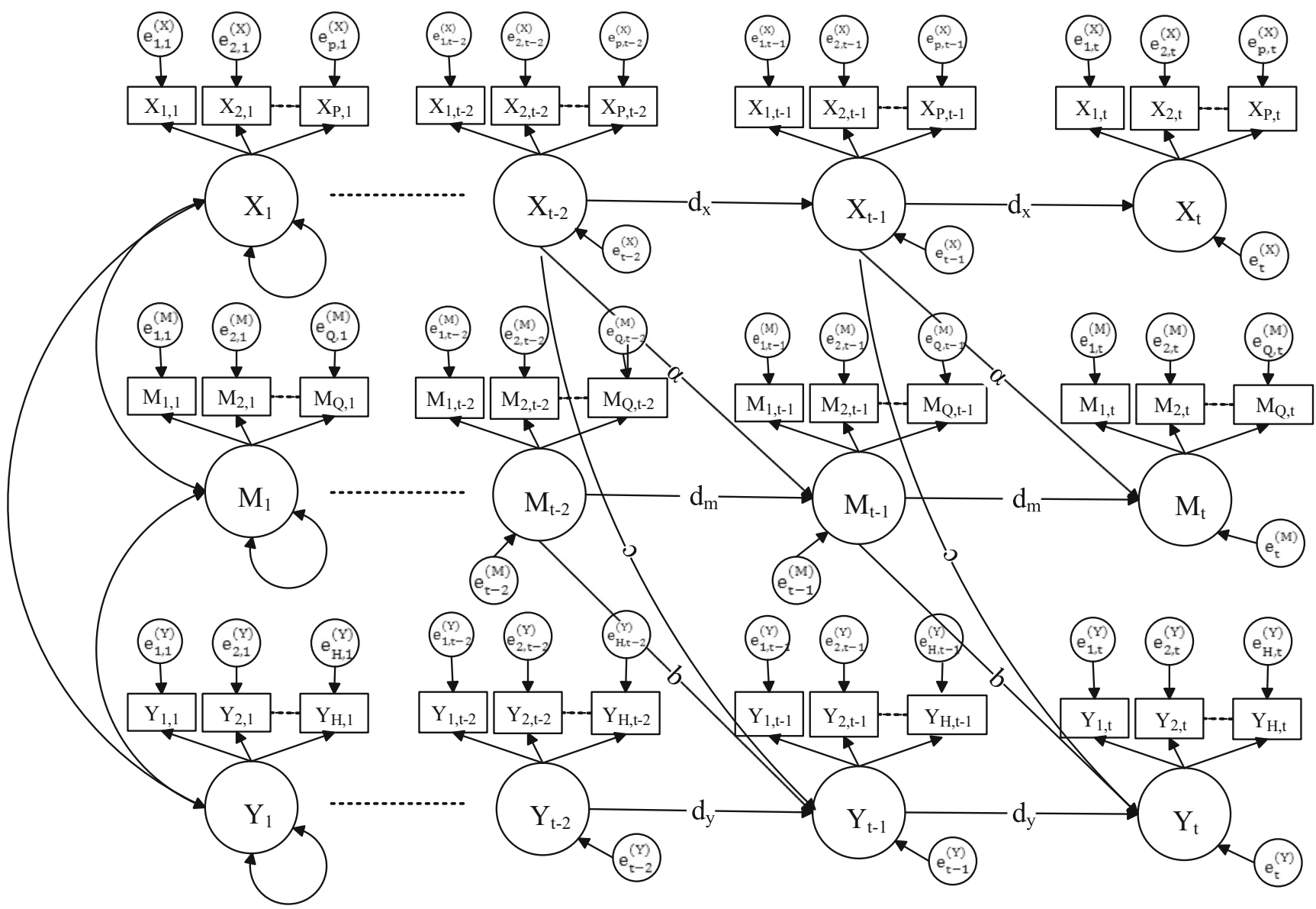

Fig. 1 Illustration of the LCPM Model. Covariances between measurement errors across time points are not shown in the figure due to the space limit

Longitudinal measurement invariance indicates that measurement properties of indicators for latent variables are time-independent. Let $\mathbf{Z}_{\mathbf{t}}$ be a vector of measured variables at time $t$, $\xi_{t}$ be a latent factor vector containing constructs intended to be measured by variables in $\mathbf{Z}_{\mathbf{t}}$ at time $t$, and $\mathbf{P}\left(\mathbf{Z}_{t} \mid \xi_{t}\right)$ be the conditional probability for scores in $\mathbf{Z}_{t}$ given scores on latent factors in $\xi_{t}$ at time $t$. Following the general definition of measurement invariance (Mellenbergh, 1989; Meredith, 1993; Meredith \& Millsap, 1992; Millsap \& Cham, 2012), measurement invariance holds if and only if $\mathbf{P}\left(\mathbf{Z}_{t} \mid \xi_{t}\right)=\mathbf{P}$ $\left(\mathbf{Z}_{t^{\prime}} \mid \xi_{t^{\prime}}\right)$ given $\xi_{t}=\xi_{t^{\prime}}$ where $t \neq t^{\prime}\left(t\right.$ and $\left.t^{\prime}=1,2, \ldots, T\right)$. This indicates that, under longitudinal measurement invariance, the conditional distributions of the measured variables are the same given the same latent factor scores on any two different occasions.

Longitudinal measurement invariance can be evaluated at four levels (Widaman, Ferrer, \& Conger, 2010): configural invariance, weak invariance (metric invariance), strong invariance (scalar invariance), and strict invariance. Configural invariance requires the factor structure be the same over time. Metric invariance, scalar invariance, and strict invariance sequentially requires equal loadings, equal intercepts, and equal measurement error variances over time. The current study focuses on direct and mediated effects among latent factors, and the mean structure is not relevant. For this reason, we do not consider the scalar invariance assumption in this study.

Most invariance studies in the literature are in the context of multiple-group analysis. A majority of these studies have focused on the effects of measurement non-invariance on between-group difference in latent means (e.g., Meredith, 1993; Sass, 2011); few studies have focused on the impact of measurement non-invariance on between-group difference in structural coefficients (e.g., Guenole \& Brown, 2014). These studies reached to the same conclusion that biased parameter estimates and inferences can occur when a reasonably large number of items were non-invariant across groups.

In contrast to the scenario with multiple groups, violation of measurement invariance has not received much attention in studying longitudinal models in which structural coefficients are of interest. Based on our review, thus far four studies have investigated the impact of longitudinal measurement noninvariance on estimating and/or testing structural coefficients in the autoregressive modeling framework. Olivera-Aguilar (2013) investigated the impact of violations of longitudinal measurement invariance on estimating and testing structural coefficients in a univariate autoregressive model with latent 
variables. In this study, all non-invariant loadings decreased over time. Liang, Yang, and Huang (2018) investigated the estimation of structural path parameters in bivariate crosslagged panel models under different conditions of longitudinal approximate invariance using Bayesian structural equation modeling. In Liang et al. (2018), two patterns of non-invariant loadings were considered: (1) all non-invariant loadings decreased over time, and (2) half non-invariant loadings decreased but the other half increased (a mixed pattern) over time. The autoregressive estimates were generally more accurate under conditions with the mixed pattern compared to those with all loadings decreasing. Gonzalez, Valente, and MacKinnon (2017) examined the consequences of violations of longitudinal measurement invariance on mediation analysis in a two-wave autoregressive mediation model when $X$ is a time-invariant binary variable denoting control vs. treatment groups.

Most recently, Zhang and Yang (in press) studied the accuracy of direct and mediated effect estimates under three CLPM models: O-CLPMs with and without a correction for measurement errors and L-CLPM. They considered only one longitudinal measurement non-invariance pattern where standardized loadings decreased by .05 over time for $2 / 3$ items of $X, M$, and $Y$. They found that the L-CLPM yielded reasonably accurate direct and mediated effect estimates. They also found that model Chi-square tests performed poorly under both invariance and non-variance conditions. Because only one specific pattern of measurement non-invariance was investigated, the results may not be generalized to models with different patterns and/or extent of measurement non-invariance. Noticing a lack of extensive study on the influence of measurement non-invariance on parameter estimates and modeldata fit under L-CLPMs, the present study investigates the impact of longitudinal measurement non-invariance on estimating and testing the direct and mediated effects in an LCLPM under a wider range of conditions.

Three major purposes of this study are: (1) to investigate how violations of longitudinal measurement invariance can impact accuracy of the direct and mediated effect estimates in L-CLPMs, (2) to examine the impact of violations of measurement invariance on the accuracy of statistical inferences regarding the direct and mediated effects, and (3) to examine whether model Chi-square statistics and fit indices can help detect longitudinal measurement non-invariance under finite samples. Specifically, we study how different patterns and extent of variations for item parameters over time can influence direct and mediated effect estimates and inferences as well as model-data fit evaluation via a simulation study.

\section{Simulation study}

This simulation study included two parts: investigation based on population covariance matrices with no influence from sampling fluctuation under different manipulated conditions, and investigation based on generated data with different levels of sample size under selected simulated conditions. Analyses based on population covariance matrices allow us to answer the first research question, while analyses based on sample data allow us to answer the second and the third research questions. The same population model was considered for both levels of investigation.

\section{The population model}

The L-CLPM shown in Fig. 1 was used as the population model in the present study. Each latent variable was measured by six items repeatedly across five occasions. Having five measurement occasions allows for investigating the impact of various patterns of measurement non-invariance in item parameters on cross-lagged panel mediation analysis. In addition, five occasions are commonly seen in longitudinal measurement invariance studies (e.g., Leite, 2007; Liang et al., 2018; McArdle, 2009; Olivera-Aguilar, 2013; Schlueter, Davidov, Schmidt, 2007; Selig \& Little, 2012). The time intervals were equal between any two adjacent occasions. The structural path coefficients were manipulated as invariant across time to keep with the stationarity assumption. Residuals of latent $X, M$, and $Y$ at any time $t$ were manipulated as uncorrelated with each other assuming no confounding variables.

Population values were selected to make variances of all latent variables measured at each occasion to be 1.0. Values of the autoregressive coefficients for latent variables were manipulated as the same across all conditions in the population model because they are not parameters of focal interest. Specifically, $d_{\mathrm{x}}=.71, d_{\mathrm{m}}=.36$, and $d_{\mathrm{y}}=.51$. The autoregressive path coefficients were chosen to be relatively large in comparison to the path coefficients among different variables. The covariances among the latent exogenous variables (i.e., $X, M$, and $Y$ measured on the first occasion) were calculated using the formulas derived by Maxwell and colleagues to keep with the equilibrium assumption (Maxwell \& Cole, 2007; Maxwell et al., 2011).

\section{Investigation based on population covariance matrices}

For the investigation based on population covariance matrices, we varied levels of seven factors to compute population covariance matrices. We did not include any conditions with longitudinal measurement invariance being satisfied. This is because the direct and indirect effects are accurate, and the model fit perfectly to the population covariance matrix when the model is correctly specified. 


\section{Design factors for computing population covariance matrices}

Proportion of non-invariant items Two levels of proportion of items violating longitudinal invariance were considered: $1 / 3$ (small) and 2/3 (large).

Role of latent factors with non-invariant items We considered violations of longitudinal invariance according to roles of latent factors, that is, predictor $(X)$, mediator $(M)$, and outcome $(Y)$. Either one, two, or three latent factors contained noninvariant items. Specifically, seven combinations of latent variables with non-invariant items were considered: $X, M, Y, X M$, $X Y, M Y$, and $X M Y$.

Type of non-invariant parameters Longitudinal noninvariance was manipulated in loadings and item residual variances to represent violations of metric invariance and strict invariance, respectively. Item intercepts and factor intercepts were fixed at zero without loss of generality.

Pattern of non-invariance As evidenced by empirical research using real data (e.g., Millsap \& Cham, 2012; Obradović, Pardini, Long, \& Loeber, 2007; Willoughby, Wirth, \& Blair, 2012), item parameters such as loadings may change monotonically or non-monotonically within an item over time, and/or change in a uniform or mixed fashion over time. To cover as many patterns of change encountered in practice as possible and meanwhile maintain the total number of conditions to a manageable limit, the following patterns of non-invariance for loadings and item residual variances were considered in the simulation: (a) a decreasing pattern in which all non-invariant item parameters decreased over time; (b) a decreasing and then increasing pattern in which all non-invariant item parameters decreased across the first half of occasions and then increased across the remaining half of occasions; and (c) a mixed pattern where half of the non-invariant item parameters decreased and the other half of the non-invariant item parameters increased over time. Variability of loadings or residual variances over time was manipulated to be the same or very similar among the three patterns.

Magnitude of non-invariance Item loadings at the first measurement occasion were fixed at .7. Similar to OliveraAguilar (2013), magnitude of metric non-invariance can be defined as the maximum difference in loadings across occasions. Specifically, a maximum difference of .1, .2, and .3 indicate small, medium, and large changes, respectively. When loadings decrease or increase over time, an item measured at the first and last time points has the maximum difference in loadings; when loadings first decrease and then increase, an item measured at the first/last and the middle time points has the maximum difference in loadings.

Loadings at the last measurement occasion can be determined by adding (for increasing change) or subtracting (for decreasing change) the defined effect sizes from loading at the first measurement occasion. Assuming equal change in loadings between adjacent occasions, values of loadings for other occasions can be determined. For the decreasing and then increasing pattern, these steps were applied to the decreasing change from the 1 st to the 3rd occasion, and then to the increasing change from the 3 rd to the 5 th occasion. Table 1 presents values of loadings manipulated under conditions with different proportions of non-invariant items, magnitude of non-invariance, and patterns of non-invariance.

Item residual variances were manipulated following a similar procedure as loadings. First, residual variances at the first measurement occasion were fixed at .5. Second, magnitude of non-invariance in residual variances was manipulated with three levels: small (a maximum difference of .1 in residual variances across occasions), medium (a maximum difference of .2), and large (a maximum difference of .3). Then, residual variances for other measurement occasions can be determined based on the effect size of non-invariance and fixed amount of change between adjacent occasions. Table 2 provides values of item residual variances manipulated under various conditions.

The current study focused on the isolated effects of different types of non-invariance parameters. Therefore, only one type of non-invariance parameter (loadings or residual variances) was investigated at a time, and conditions with combinations of these two types of noninvariance parameters were not considered. Under the non-invariant loading conditions, item residual variances were manipulated as invariant and fixed at .5 across time; under the non-invariant item residual variance conditions, factor loadings were manipulated as invariant and fixed at .7 across time. Based on the manipulated loadings and item residual variances, the value of standardized loadings for items was from .492 to .843 across all conditions.

Size of the mediated effect Based on Cohen's criteria for incremental effects $f^{2}$ (.02 for small, .15 for medium, and .35 for large; Cohen, 1988), the value of .3 was selected for the $a$ and $b$ path coefficients to generate medium to large incremental effects. Under the non-zero mediated effect conditions, values of the $a$ and $b$ paths were set to be equal ( $a=b$ $=.3)$ and the resulting population mediated effect was $.09(.3 \times$ .3). Three conditions of zero mediated effect were also investigated: $a=b=0, a=.3$ and $b=0$, or $a=0$ and $b=.3$.

Size of the direct effect The direct path coefficient $c^{\prime}$ was 0 or .15. The latter effect size represented a medium to large 
Table 1. Manipulated values of factor loadings for data generation

\begin{tabular}{|c|c|c|c|c|c|c|c|c|c|c|c|c|c|c|c|}
\hline \multirow[t]{3}{*}{ Pattern } & \multirow[t]{3}{*}{ Proportion } & \multirow[t]{3}{*}{ Item } & \multicolumn{13}{|c|}{ Magnitude of non-invariance } \\
\hline & & & \multicolumn{5}{|c|}{ Small } & \multicolumn{4}{|c|}{ Medium } & \multicolumn{4}{|c|}{ Large } \\
\hline & & & $T 1$ & $T 2$ & $T 3$ & $T 4$ & $T 5$ & $T 2$ & $T 3$ & $T 4$ & $T 5$ & $T 2$ & $T 3$ & $T 4$ & T5 \\
\hline \multirow[t]{12}{*}{ Decreasing } & \multirow[t]{6}{*}{$1 / 3$} & 1 & .7 & .675 & .65 & .625 & .6 & .65 & .6 & .55 & .5 & .625 & .55 & .475 & .4 \\
\hline & & 2 & .7 & .675 & .65 & .625 & .6 & .65 & .6 & .55 & .5 & .625 & .55 & .475 & .4 \\
\hline & & 3 & .7 & .7 & .7 & .7 & .7 & .7 & .7 & .7 & .7 & .7 & .7 & .7 & .7 \\
\hline & & 4 & .7 & .7 & .7 & .7 & .7 & .7 & .7 & .7 & .7 & .7 & .7 & .7 & .7 \\
\hline & & 5 & .7 & .7 & .7 & .7 & .7 & .7 & .7 & .7 & .7 & .7 & .7 & .7 & .7 \\
\hline & & 6 & .7 & .7 & .7 & .7 & .7 & .7 & .7 & .7 & .7 & .7 & .7 & .7 & .7 \\
\hline & \multirow[t]{6}{*}{$2 / 3$} & 1 & .7 & .675 & .65 & .625 & .6 & .65 & .6 & .55 & .5 & .625 & .55 & .475 & .4 \\
\hline & & 2 & .7 & .675 & .65 & .625 & .6 & .65 & .6 & .55 & .5 & .625 & .55 & .475 & .4 \\
\hline & & 3 & .7 & .675 & .65 & .625 & .6 & .65 & .6 & .55 & .5 & .625 & .55 & .475 & .4 \\
\hline & & 4 & .7 & .675 & .65 & .625 & .6 & .65 & .6 & .55 & .5 & .625 & .55 & .475 & .4 \\
\hline & & 5 & .7 & .7 & .7 & .7 & .7 & .7 & .7 & .7 & .7 & .7 & .7 & .7 & .7 \\
\hline & & 6 & .7 & .7 & .7 & .7 & .7 & .7 & .7 & .7 & .7 & .7 & .7 & .7 & .7 \\
\hline \multirow{12}{*}{$\begin{array}{l}\text { Decreasing } \\
\text { and then increasing }\end{array}$} & \multirow[t]{6}{*}{$1 / 3$} & 1 & .7 & .65 & .6 & .65 & .7 & .6 & .5 & .6 & .7 & .55 & .4 & .55 & .7 \\
\hline & & 2 & .7 & .65 & .6 & .65 & .7 & .6 & .5 & .6 & .7 & .55 & .4 & .55 & .7 \\
\hline & & 3 & .7 & .7 & .7 & .7 & .7 & .7 & .7 & .7 & .7 & .7 & .7 & .7 & .7 \\
\hline & & 4 & .7 & .7 & .7 & .7 & .7 & .7 & .7 & .7 & .7 & .7 & .7 & .7 & .7 \\
\hline & & 5 & .7 & .7 & .7 & .7 & .7 & .7 & .7 & .7 & .7 & .7 & .7 & .7 & .7 \\
\hline & & 6 & .7 & .7 & .7 & .7 & .7 & .7 & .7 & .7 & .7 & .7 & .7 & .7 & .7 \\
\hline & \multirow[t]{6}{*}{$2 / 3$} & 1 & .7 & .65 & .6 & .65 & .7 & .6 & .5 & .6 & .7 & .55 & .4 & .55 & .7 \\
\hline & & 2 & .7 & .65 & .6 & .65 & .7 & .6 & .5 & .6 & .7 & .55 & .4 & .55 & .7 \\
\hline & & 3 & .7 & .65 & .6 & .65 & .7 & .6 & .5 & .6 & .7 & .55 & .4 & .55 & .7 \\
\hline & & 4 & .7 & .65 & .6 & .65 & .7 & .6 & .5 & .6 & .7 & .55 & .4 & .55 & .7 \\
\hline & & 5 & .7 & .7 & .7 & .7 & .7 & .7 & .7 & .7 & .7 & .7 & .7 & .7 & .7 \\
\hline & & 6 & .7 & .7 & .7 & .7 & .7 & .7 & .7 & .7 & .7 & .7 & .7 & .7 & .7 \\
\hline \multirow[t]{12}{*}{ Mixed } & \multirow[t]{6}{*}{$1 / 3$} & 1 & .7 & .675 & .65 & .625 & .6 & .65 & .6 & .55 & .5 & .625 & .55 & .475 & .4 \\
\hline & & 2 & .7 & .725 & .75 & .775 & .8 & .75 & .8 & .85 & .9 & .775 & .85 & .925 & 1 \\
\hline & & 3 & .7 & .7 & .7 & .7 & .7 & .7 & .7 & .7 & .7 & .7 & .7 & .7 & .7 \\
\hline & & 4 & .7 & .7 & .7 & .7 & .7 & .7 & .7 & .7 & .7 & .7 & .7 & .7 & .7 \\
\hline & & 5 & .7 & .7 & .7 & .7 & .7 & .7 & .7 & .7 & .7 & .7 & .7 & .7 & .7 \\
\hline & & 6 & .7 & .7 & .7 & .7 & .7 & .7 & .7 & .7 & .7 & .7 & .7 & .7 & .7 \\
\hline & \multirow[t]{6}{*}{$2 / 3$} & 1 & .7 & .675 & .65 & .625 & .6 & .65 & .6 & .55 & .5 & .625 & .55 & .475 & .4 \\
\hline & & 2 & .7 & .675 & .65 & .625 & .6 & .65 & .6 & .55 & .5 & .625 & .55 & .475 & .4 \\
\hline & & 3 & .7 & .725 & .75 & .775 & .8 & .75 & .8 & .85 & .9 & .775 & .85 & .925 & 1 \\
\hline & & 4 & .7 & .725 & .75 & .775 & .8 & .75 & .8 & .85 & .9 & .775 & .85 & .925 & 1 \\
\hline & & 5 & .7 & .7 & .7 & .7 & .7 & .7 & .7 & .7 & .7 & .7 & .7 & .7 & .7 \\
\hline & & 6 & .7 & .7 & .7 & .7 & .7 & .7 & .7 & .7 & .7 & .7 & .7 & .7 & .7 \\
\hline
\end{tabular}

incremental effect $f^{2}$, when the predictor $X$ was added into the model for $Y$.

In total, 2016 covariance matrices were obtained: 2 proportions of non-invariant items $\times 7$ combinations of latent variables with non-invariant items $\times 3$ patterns of non- invariance $\times 2$ types of non-invariant parameters $\times 3$ magnitude of non-invariance $\times 4$ sizes of the $a$ and $b$ paths for the mediated effect $\times 2$ sizes of direct effects. Measurement errors across time were assumed to be uncorrelated with each other under the investigated conditions. 
Table 2. Manipulated values of item residual variances for data generation

\begin{tabular}{|c|c|c|c|c|c|c|c|c|c|c|c|c|c|c|c|}
\hline \multirow[t]{3}{*}{ Pattern } & \multirow[t]{3}{*}{ Proportion } & \multirow[t]{3}{*}{ Item } & \multicolumn{13}{|c|}{ Magnitude of non-invariance } \\
\hline & & & \multicolumn{5}{|c|}{ Small } & \multicolumn{4}{|c|}{ Medium } & \multicolumn{4}{|c|}{ Large } \\
\hline & & & $T 1$ & $T 2$ & $T 3$ & $T 4$ & $T 5$ & $T 2$ & $T 3$ & $T 4$ & $T 5$ & $T 2$ & $T 3$ & $T 4$ & T5 \\
\hline \multirow[t]{12}{*}{ Decreasing } & \multirow[t]{6}{*}{$1 / 3$} & 1 & .5 & .475 & .45 & .425 & .4 & .45 & .4 & .35 & .3 & .425 & .35 & .275 & .2 \\
\hline & & 2 & .5 & .475 & .45 & .425 & .4 & .45 & .4 & .35 & .3 & .425 & .35 & .275 & .2 \\
\hline & & 3 & .5 & .5 & .5 & .5 & .5 & .5 & .5 & .5 & .5 & .5 & .5 & .5 & .5 \\
\hline & & 4 & .5 & .5 & .5 & .5 & .5 & .5 & .5 & .5 & .5 & .5 & .5 & .5 & .5 \\
\hline & & 5 & .5 & .5 & .5 & .5 & .5 & .5 & .5 & .5 & .5 & .5 & .5 & .5 & .5 \\
\hline & & 6 & .5 & .5 & .5 & .5 & .5 & .5 & .5 & .5 & .5 & .5 & .5 & .5 & .5 \\
\hline & \multirow[t]{6}{*}{$2 / 3$} & 1 & .5 & .475 & .45 & .425 & .4 & .45 & .4 & .35 & .3 & .425 & .35 & .275 & .2 \\
\hline & & 2 & .5 & .475 & .45 & .425 & .4 & .45 & .4 & .35 & .3 & .425 & .35 & .275 & .2 \\
\hline & & 3 & .5 & .475 & .45 & .425 & .4 & .45 & .4 & .35 & .3 & .425 & .35 & .275 & .2 \\
\hline & & 4 & .5 & .475 & .45 & .425 & .4 & .45 & .4 & .35 & .3 & .425 & .35 & .275 & .2 \\
\hline & & 5 & .5 & .5 & .5 & .5 & .5 & .5 & .5 & .5 & .5 & .5 & .5 & .5 & .5 \\
\hline & & 6 & .5 & .5 & .5 & .5 & .5 & .5 & .5 & .5 & .5 & .5 & .5 & .5 & .5 \\
\hline \multirow{12}{*}{$\begin{array}{l}\text { Decreasing } \\
\text { and then increasing }\end{array}$} & \multirow[t]{6}{*}{$1 / 3$} & 1 & .5 & .45 & .4 & .45 & .5 & .4 & .3 & .4 & .5 & .35 & .2 & .35 & .5 \\
\hline & & 2 & .5 & .45 & .4 & .45 & .5 & .4 & .3 & .4 & .5 & .35 & .2 & .35 & .5 \\
\hline & & 3 & .5 & .5 & .5 & .5 & .5 & .5 & .5 & .5 & .5 & .5 & .5 & .5 & .5 \\
\hline & & 4 & .5 & .5 & .5 & .5 & .5 & .5 & .5 & .5 & .5 & .5 & .5 & .5 & .5 \\
\hline & & 5 & .5 & .5 & .5 & .5 & .5 & .5 & .5 & .5 & .5 & .5 & .5 & .5 & .5 \\
\hline & & 6 & .5 & .5 & .5 & .5 & .5 & .5 & .5 & .5 & .5 & .5 & .5 & .5 & .5 \\
\hline & \multirow[t]{6}{*}{$2 / 3$} & 1 & .5 & .45 & .4 & .45 & .5 & .4 & .3 & .4 & .5 & .35 & .2 & .35 & .5 \\
\hline & & 2 & .5 & .45 & .4 & .45 & .5 & .4 & .3 & .4 & .5 & .35 & .2 & .35 & .5 \\
\hline & & 3 & .5 & .45 & .4 & .45 & .5 & .4 & .3 & .4 & .5 & .35 & .2 & .35 & .5 \\
\hline & & 4 & .5 & .45 & .4 & .45 & .5 & .4 & .3 & .4 & .5 & .35 & .2 & .35 & .5 \\
\hline & & 5 & .5 & .5 & .5 & .5 & .5 & .5 & .5 & .5 & .5 & .5 & .5 & .5 & .5 \\
\hline & & 6 & .5 & .5 & .5 & .5 & .5 & .5 & .5 & .5 & .5 & .5 & .5 & .5 & .5 \\
\hline \multirow[t]{12}{*}{ Mixed } & \multirow[t]{6}{*}{$1 / 3$} & 1 & .5 & .475 & .45 & .425 & .4 & .45 & .4 & .35 & .3 & .425 & .35 & .275 & .2 \\
\hline & & 2 & .5 & .525 & .55 & .575 & .6 & .55 & .6 & .65 & .7 & .575 & .65 & .725 & .8 \\
\hline & & 3 & .5 & .5 & .5 & .5 & .5 & .5 & .5 & .5 & .5 & .5 & .5 & .5 & .5 \\
\hline & & 4 & .5 & .5 & .5 & .5 & .5 & .5 & .5 & .5 & .5 & .5 & .5 & .5 & .5 \\
\hline & & 5 & .5 & .5 & .5 & .5 & .5 & .5 & .5 & .5 & .5 & .5 & .5 & .5 & .5 \\
\hline & & 6 & .5 & .5 & .5 & .5 & .5 & .5 & .5 & .5 & .5 & .5 & .5 & .5 & .5 \\
\hline & \multirow[t]{6}{*}{$2 / 3$} & 1 & .5 & .475 & .45 & .425 & .4 & .45 & .4 & .35 & .3 & .425 & .35 & .275 & .2 \\
\hline & & 2 & .5 & .475 & .45 & .425 & .4 & .45 & .4 & .35 & .3 & .425 & .35 & .275 & .2 \\
\hline & & 3 & .5 & .525 & .55 & .575 & .6 & .55 & .6 & .65 & .7 & .575 & .65 & .725 & .8 \\
\hline & & 4 & .5 & .525 & .55 & .575 & .6 & .55 & .6 & .65 & .7 & .575 & .65 & .725 & .8 \\
\hline & & 5 & .5 & .5 & .5 & .5 & .5 & .5 & .5 & .5 & .5 & .5 & .5 & .5 & .5 \\
\hline & & 6 & .5 & .5 & .5 & .5 & .5 & .5 & .5 & .5 & .5 & .5 & .5 & .5 & .5 \\
\hline
\end{tabular}

\section{Fitted models}

Each population covariance matrix was fitted by a constrained model to keep with either the metric or strict invariance assumption. To be specific, (1) under non-invariant loadings conditions, equality constraints were imposed on loadings across time in the fitted model, leaving the item residual variances freely estimated to keep with the metric invariance 
assumption (loading-constrained model); and (2) under noninvariant residual variances conditions, equality constraints were put on both loadings and item residual variances across time in the fitted model to keep with the strict invariance assumption (loading- and variance-constrained model) ${ }^{3}$.

In addition, the following constraints were imposed to fitted models across all conditions: (1) the structural path coefficients $\left(d_{\mathrm{x}}, d_{\mathrm{m}}, d_{\mathrm{y}}, a, b\right.$, and $\left.c^{\prime}\right)$ were constrained to be equal over time; (2) factor residual covariances were fixed at zero; (3) factor residual variances were constrained to be equal starting from the second time point ${ }^{4}$; and (4) loadings of the last item for each latent variable at each measurement occasion were fixed at its population value (i.e., .7) for identification purpose. Because the estimated structural paths were the same across different time points in analysis models, the estimated mediated effect from $X_{1}-M_{2}-Y_{3}, X_{2}-M_{3}-Y_{4}$, and $X_{3}-M_{4^{-}}$ $Y_{5}$ was the same, and was calculated as the product of the $a$ and $b$ path coefficients under each condition. All models were analyzed in Mplus version 7 (Muthén \& Muthén, 1998-2012) using the maximum likelihood (ML) estimation method. When fitting each population covariance matrix to the LCLPM in Mplus, number of observations (i.e., sample size) has to be provided. The choice of size does not impact the model fit function and estimates for population investigation. We chose $N=1000$, the largest sample size considered in the second part of the simulation study.

\section{Results based on analyses of population covariance matrices}

In general, longitudinal non-invariance in item residual variances resulted in negligible deviations between parameter estimates and population parameters under loading- and variance-constrained models. Mean estimates for $a, b, a b$, and $c^{\prime}$ based on the loading- and variance- constrained models with non-invariant residual variances and non-zero $a, b$, and $c^{\prime}$ ( $a=b=.3$ and $c^{\prime}=.15$ ) are shown in Figs. 2, 3, 4, and 5. Conditions with other values of $a, b$, and $c$ showed similar result patterns.

In contrast, many conditions with non-invariant loadings can result in noticeable deviations between parameter estimates and population parameters under loading-constrained

\footnotetext{
${ }^{3}$ Full invariance models were used in the present study. In practice, partial invariance models are sometimes specified allowing time-varying parameter estimates for some items (e.g., Obradović, et al., 2007; Willoughby, Wirth \& Blair, 2012). We expect that effect estimates would be very close to the population values when fitting the partial invariance models to data because the fitted models are consistent with the data generation models. More discussions on partially invariant models can be found in the Discussion and conclusions section.

${ }^{4}$ This is to keep with the equilibrium assumption such that covariance matrices of latent $X, M$, and $Y$ were invariant starting from the second time point. We regard the latent $X, M$, and $Y$ at the first time as fixed and therefore did not fix their covariances to be the same as those from other time points.
}

models. Therefore, we focus on results when metric invariance was violated (i.e., loading-constrained models). When the true effects were zero, the estimated direct and mediated effects based on the loading-constrained model were zero or very close to zero across all non-invariance conditions; when the true effect was non-zero, differences can be observed between the estimated direct and mediated effects and the true effects under most conditions.

For example, Figs. 6, 7, 8, and 9 show mean estimates for $a, b, a b$, and $c^{\prime}$ respectively based on the loading-constrained model with non-invariant loadings and non-zero $a, b$, and $c^{\prime}(a$ $=b=.3$ and $c^{\prime}=.15$ ). For estimates of $a$ and $b$ (Figs. 6 and 7), it can be noticed that non-invariance in items measuring $Y$ only had minimal impact on estimates of $a$, while non-invariance in items measuring $X$ only had minimal effect on estimates of $b$. Furthermore, with decreasing loadings, estimates for $a$ and $b$ were both smaller than their true values; in contrast, with decreasing and then increasing loadings, the estimates of $a$ and $b$ were in opposite patterns (i.e., one increasing and the other decreasing) or were close to their true values. This made estimates of $a b$ similar to its true value. Estimates of $a$ and $b$ were in general accurate when half loadings increased, and the other half decreased over time (the mixed pattern). Therefore, overall, the decreasing pattern produced less accurate direct and mediated effect estimates compared to the other two patterns. Furthermore, as magnitude of non-invariance, proportion of non-invariant items, and/or number of latent variables with non-invariant items increased in the model, direct and mediated effect estimates tended to be less accurate.

Table 3 summarizes conditions under which differences between the direct and mediated effect estimates and the corresponding true values were relatively large (i.e., at least $5 \%$ ) when the metric invariance assumption was violated. ${ }^{5}$ Some of these conditions were further examined under finite samples with different levels of sample size.

\footnotetext{
${ }^{5}$ To increase generalizability of the results, additional population covariance matrices were computed under the following seven conditions with different sign of the $a$ - and $b$-paths and with increasing loading pattern. These seven conditions had large magnitude of non-invariance and large proportion (2/3) of non-invariant items measuring $X, M$ and $Y$. Specifically, (1) decreasing loading, $a=.3, b=-.3$, and $c^{\prime}=.15$, (2) decreasing loading, $a=-.3, b=.3$, and $c^{\prime}=$ .15 , (3) decreasing loading, $a=-.3, b=-.3$, and $c^{\prime}=.15$, (4) increasing loading, $a=.3, b=-.3$, and $c^{\prime}=.15$, (5) increasing loading, $a=-.3, b=.3$, and $c^{\prime}=.15$, (6) increasing loading, $a=.3, b=.3$, and $c^{\prime}=.15$, and (7) increasing loading, $a=-.3, b=-.3$, and $c^{\prime}=.15$. Results showed that the direct and mediated effect estimates (absolute values of mediated effect estimates when $a$ and $b$ had opposite signs) were underestimated in the decreasing loading conditions, while they were overestimated in the increasing loading conditions. The sign of the $a$ and $b$ paths determined the sign of the mediated effect. However, it did not appear to impact on the magnitude of deviations from the population values and model-data fit across the investigated conditions. These findings indicated that results from this simulation study can be generalized to scenarios with different signs of structural paths.
} 


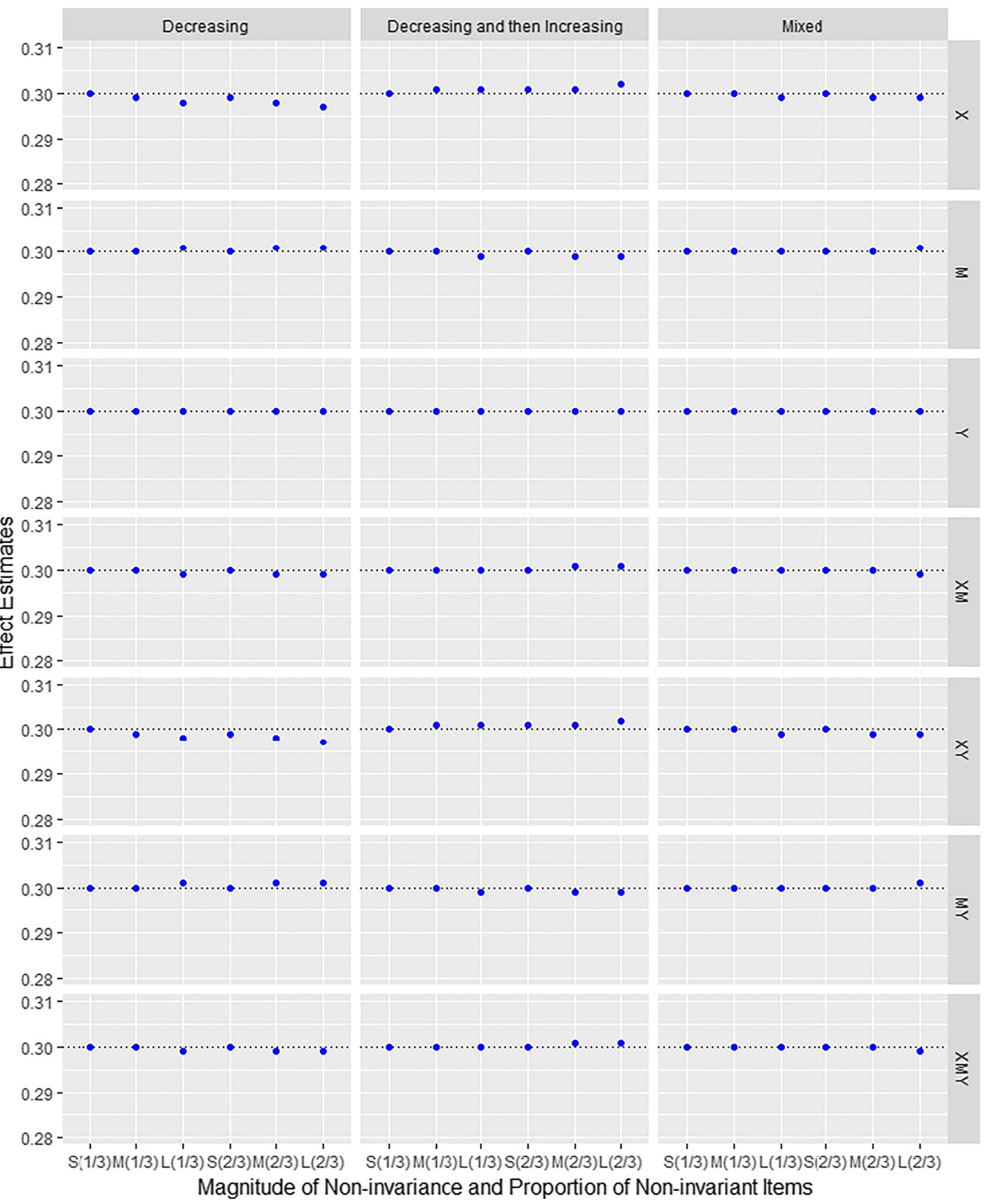

Fig. 2 Estimates of parameter $a$ under the non-invariant item residual variance conditions with partial mediation $\left(a=b=.3\right.$ and $\left.c^{\prime}=.15\right) . X$, $M, Y, X M, X Y, M Y$, and $X M Y$ denote the role of latent variables with noninvariant item parameters playing in the model; S, M, and L indicate

small, medium, and large magnitude of non-invariance, respectively; 1/ 3 and $2 / 3$ indicate small and large proportion of non-invariant items, respectively 


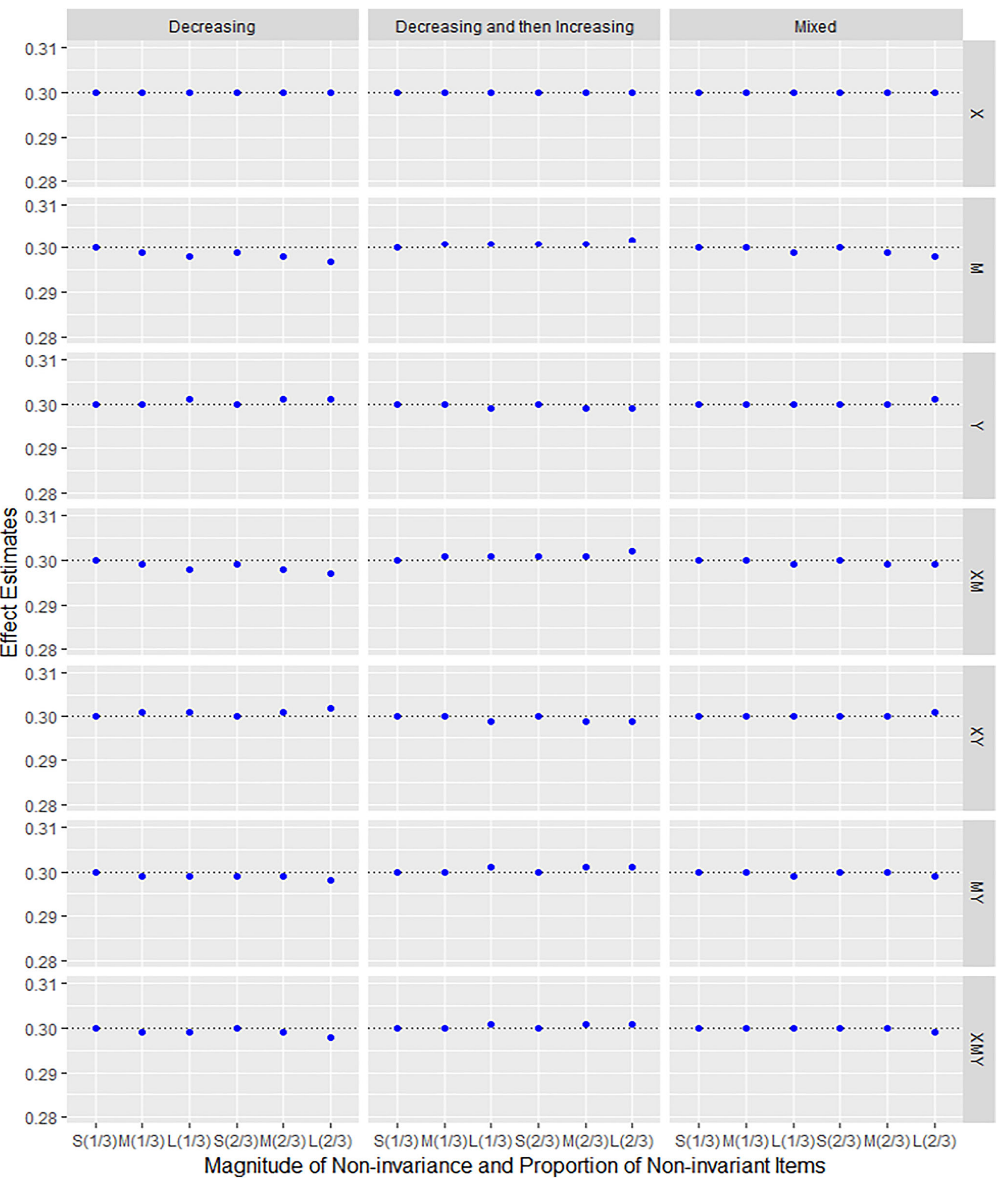

Fig. 3 Estimates of parameter $b$ under the non-invariant item residual variance conditions with partial mediation $\left(a=b=.3\right.$ and $\left.c^{\prime}=.15\right) . X$, $M, Y, X M, X Y, M Y$, and $X M Y$ denote the role of latent variables with noninvariant item parameters playing in the model; S, M, and L indicate

small, medium, and large magnitude of non-invariance, respectively; 1/ 3 and 2/3 indicate small and large proportion of non-invariant items, respectively 


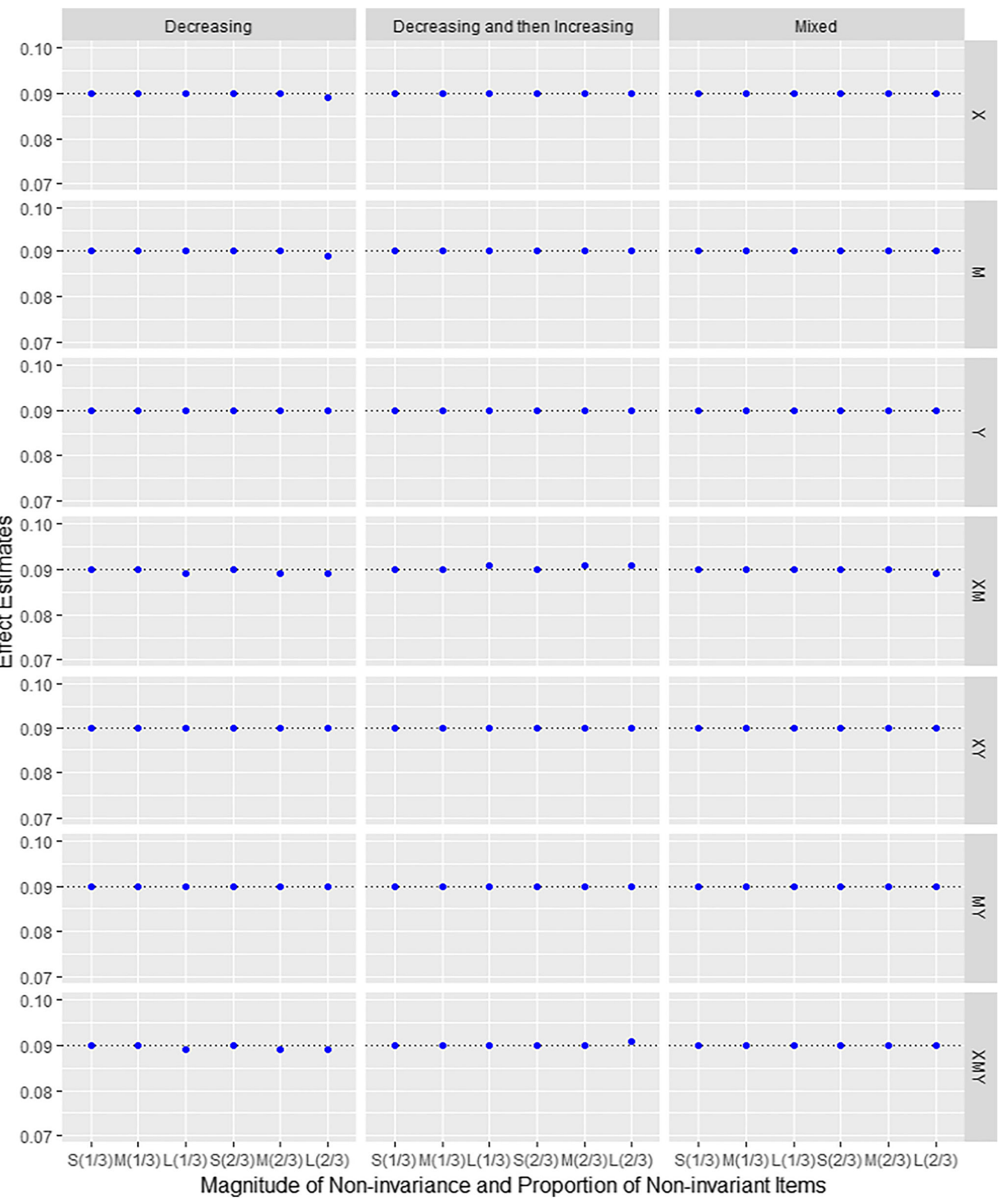

Fig. 4 Mediated effect under the non-invariant item residual variance conditions with partial mediation $\left(a=b=.3\right.$ and $\left.c^{\prime}=.15\right) . X, M, Y, X M$,

item parameters playing in the model; S, M, and L indicate small, medi$X Y, M Y$, and $X M Y$ denote the role of latent variables with non-invariant $\mathrm{um}$, and large magnitude of non-invariance, respectively; $1 / 3$ and $2 / 3$ indicate small and large proportion of non-invariant items, respectively 


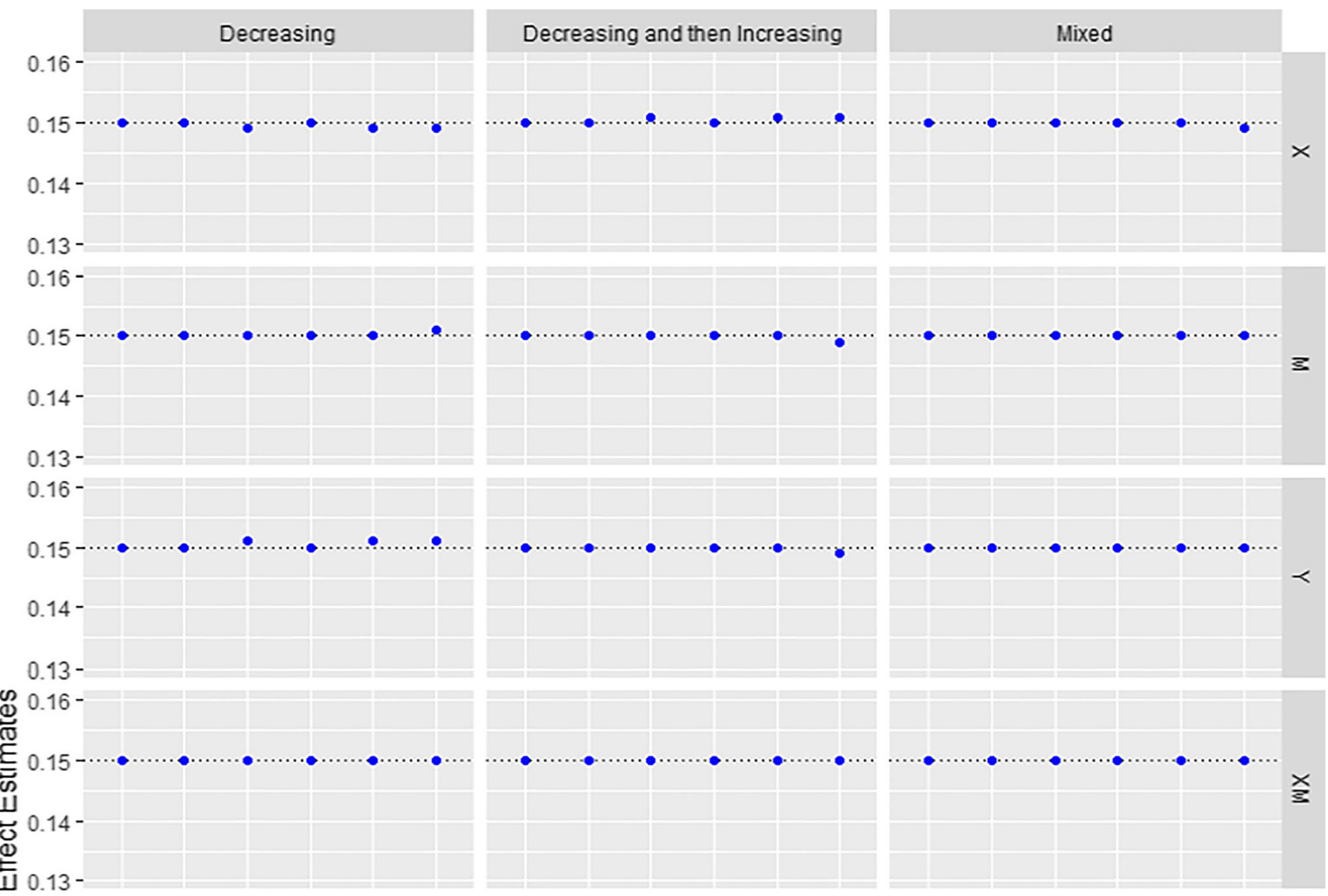

0.16 -

$0.15-\cdots$
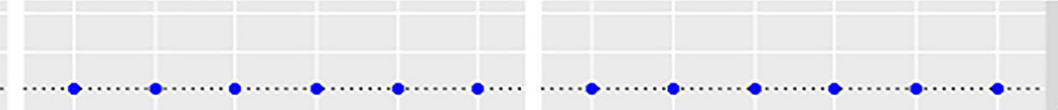

$0.14-$

0.13 -

0.16 -

$0.15-\cdots$

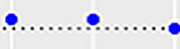

$0.14-$

0.13 -

0.16 -

$0.15-\cdots$

0.14 -

$0.13-$ $\mathrm{S}(1 / 3) \mathrm{M}^{\prime}(1 / 3) \mathrm{L}(1 / 3) \mathrm{S}(2 / 3) \mathrm{M}(2 / 3) \mathrm{L}(2 / 3) \quad \mathrm{S}(1 / 3) \mathrm{M}(1 / 3) \mathrm{L}(1 / 3) \mathrm{S}(2 / 3) \mathrm{M}(2 / 3) \mathrm{L}(2 / 3) \quad \mathrm{S}(1 / 3) \mathrm{M}(1 / 3) \mathrm{L}(1 / 3) \mathrm{S}(2 / 3) \mathrm{M}(2 / 3) \mathrm{L}(2 / 3)$

\section{Magnitude of Non-invariance and Proportion of Non-invariant ltems}

Fig. 5 Direct effect under the non-invariant item residual variance conditions with partial mediation $\left(a=b=.3\right.$ and $\left.c^{\prime}=.15\right) . X, M, Y, X M, X Y$, $M Y$, and $X M Y$ denote the role of latent variables with non-invariant item parameters playing in the model; $\mathrm{S}, \mathrm{M}$, and $\mathrm{L}$ indicate small, medium, and large magnitude of non-invariance, respectively; $1 / 3$ and $2 / 3$ indicate small and large proportion of non-invariant items, respectively 


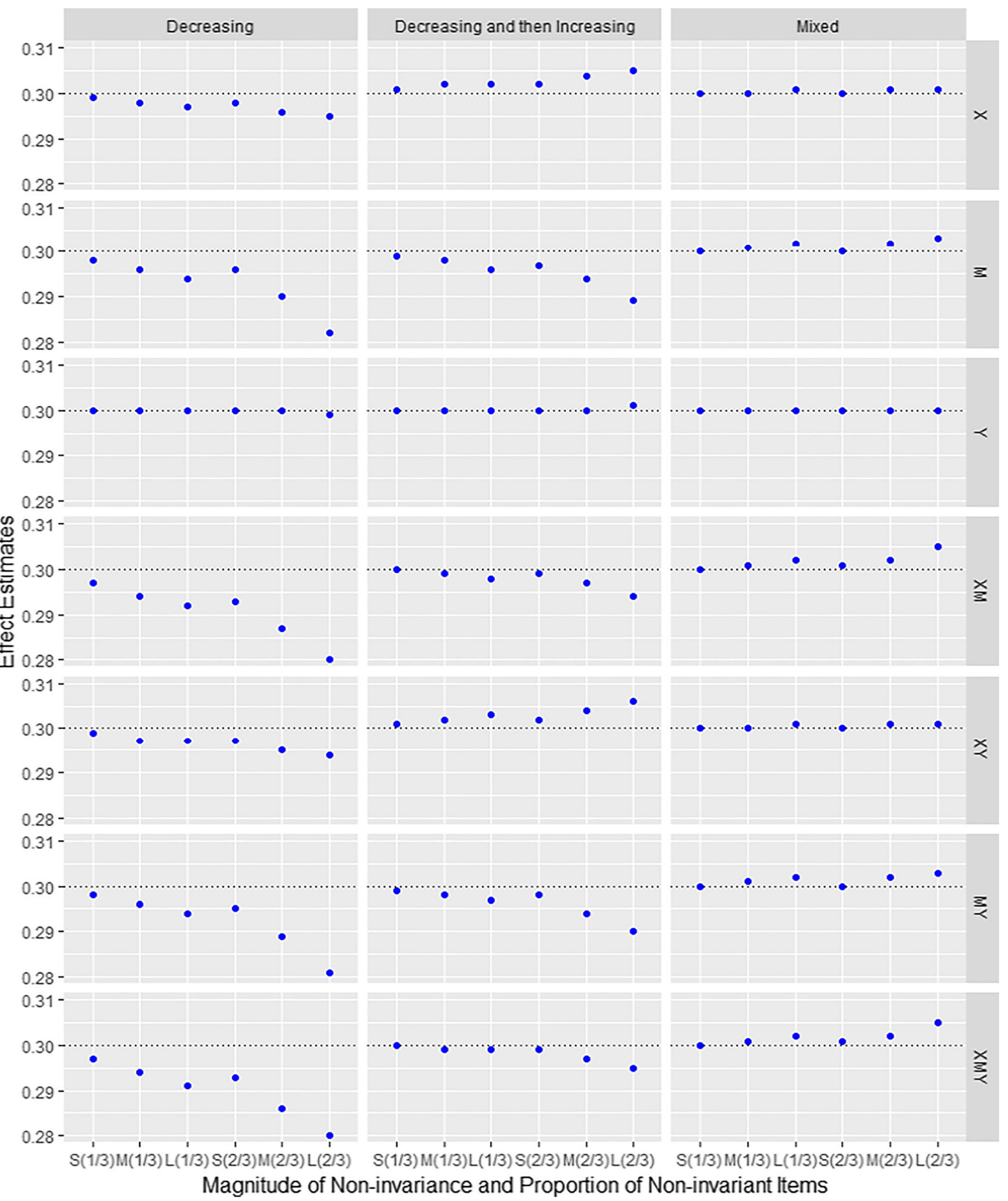

Fig. 6 Estimates of parameter $a$ under the non-invariant loading conditions with partial mediation ( $a=b=.3$ and $\left.c^{\prime}=.15\right) . X, M, Y, X M, X Y, M Y$,

parameters playing in the model; $\mathrm{S}, \mathrm{M}$, and $\mathrm{L}$ indicate small, medium, and $X M Y$ denote the role of latent variables with non-invariant item and large magnitude of non-invariance, respectively; $1 / 3$ and $2 / 3$ indicate small and large proportion of non-invariant items, respectively 


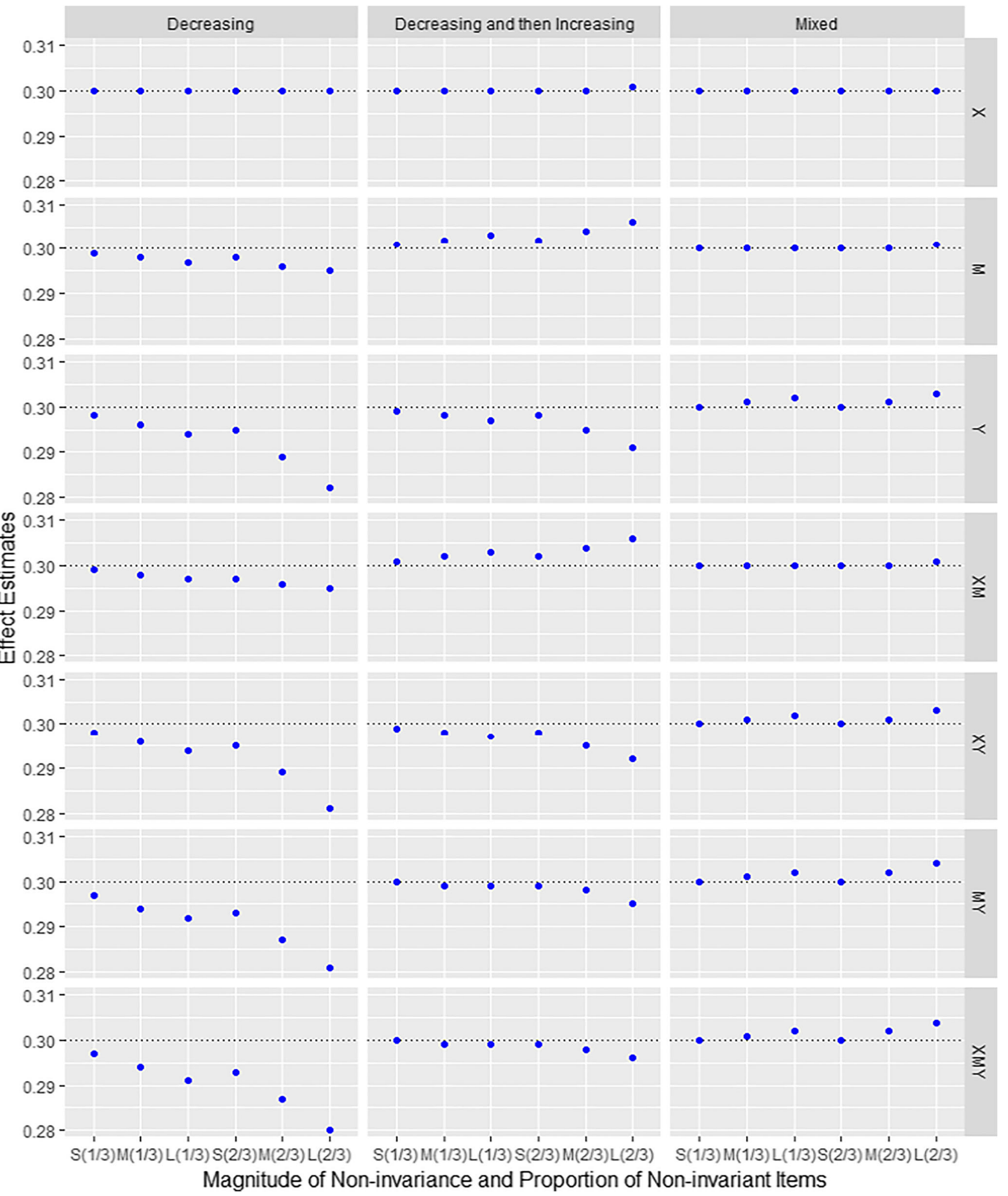

Fig. 7 Estimates of parameter $b$ under the non-invariant loading conditions with partial mediation $\left(a=b=.3\right.$ and $\left.c^{\prime}=.15\right) . X, M, Y, X M, X Y, M Y$,

parameters playing in the model; $\mathrm{S}, \mathrm{M}$, and $\mathrm{L}$ indicate small, medium, and $X M Y$ denote the role of latent variables with non-invariant item and large magnitude of non-invariance, respectively; $1 / 3$ and $2 / 3$ indicate small and large proportion of non-invariant items, respectively 


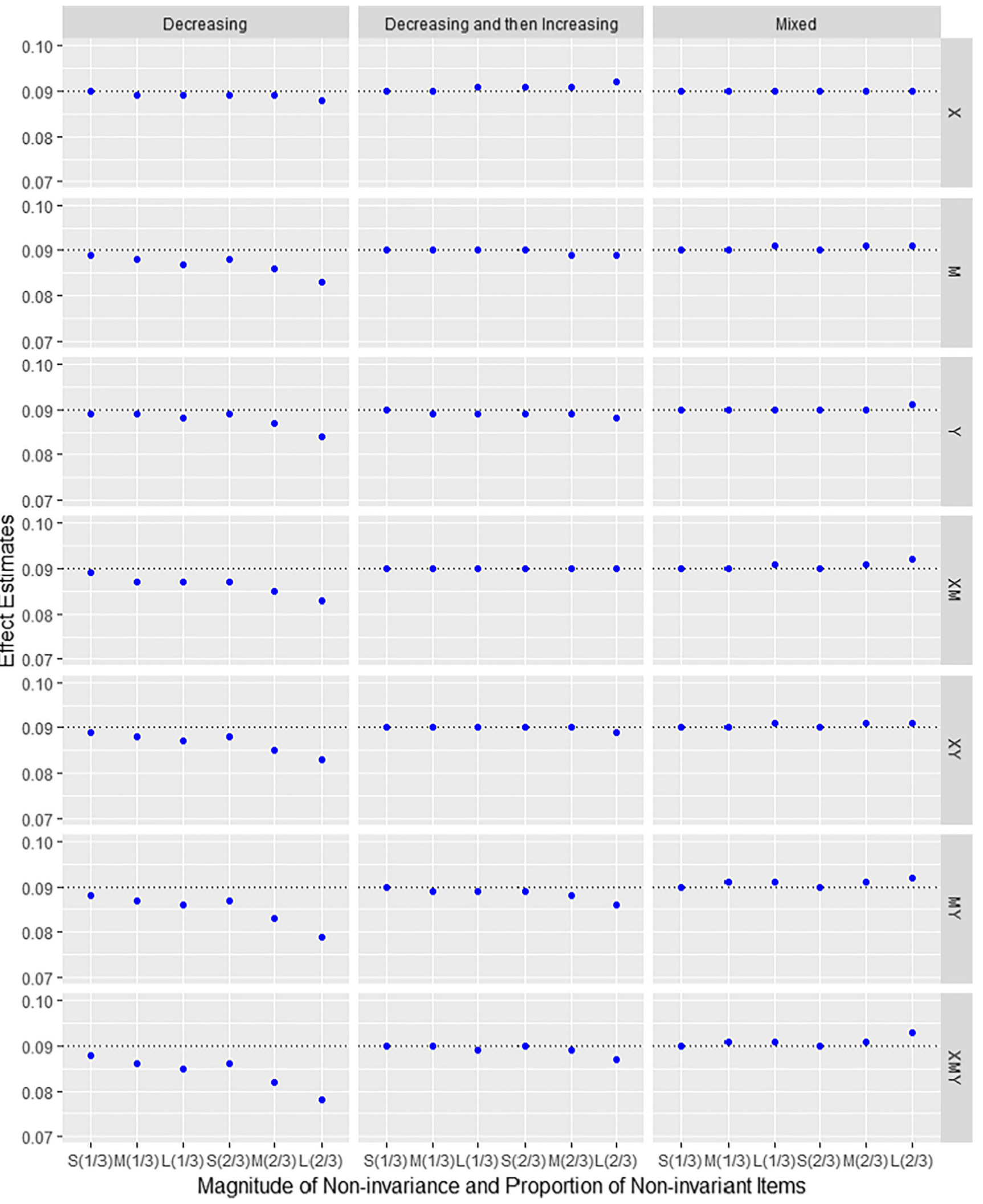

Fig. 8 Mediated effect under the non-invariant loading conditions with partial mediation ( $a=b=.3$ and $c^{\prime}=.15$ ). $X, M, Y, X M, X Y, M Y$, and $X M Y$ denote the role of latent variables with non-invariant item parameters playing in the model; $\mathrm{S}, \mathrm{M}$, and $\mathrm{L}$ indicate small, medium, and large magnitude of non-invariance, respectively; $1 / 3$ and $2 / 3$ indicate small and large proportion of non-invariant items, respectively 


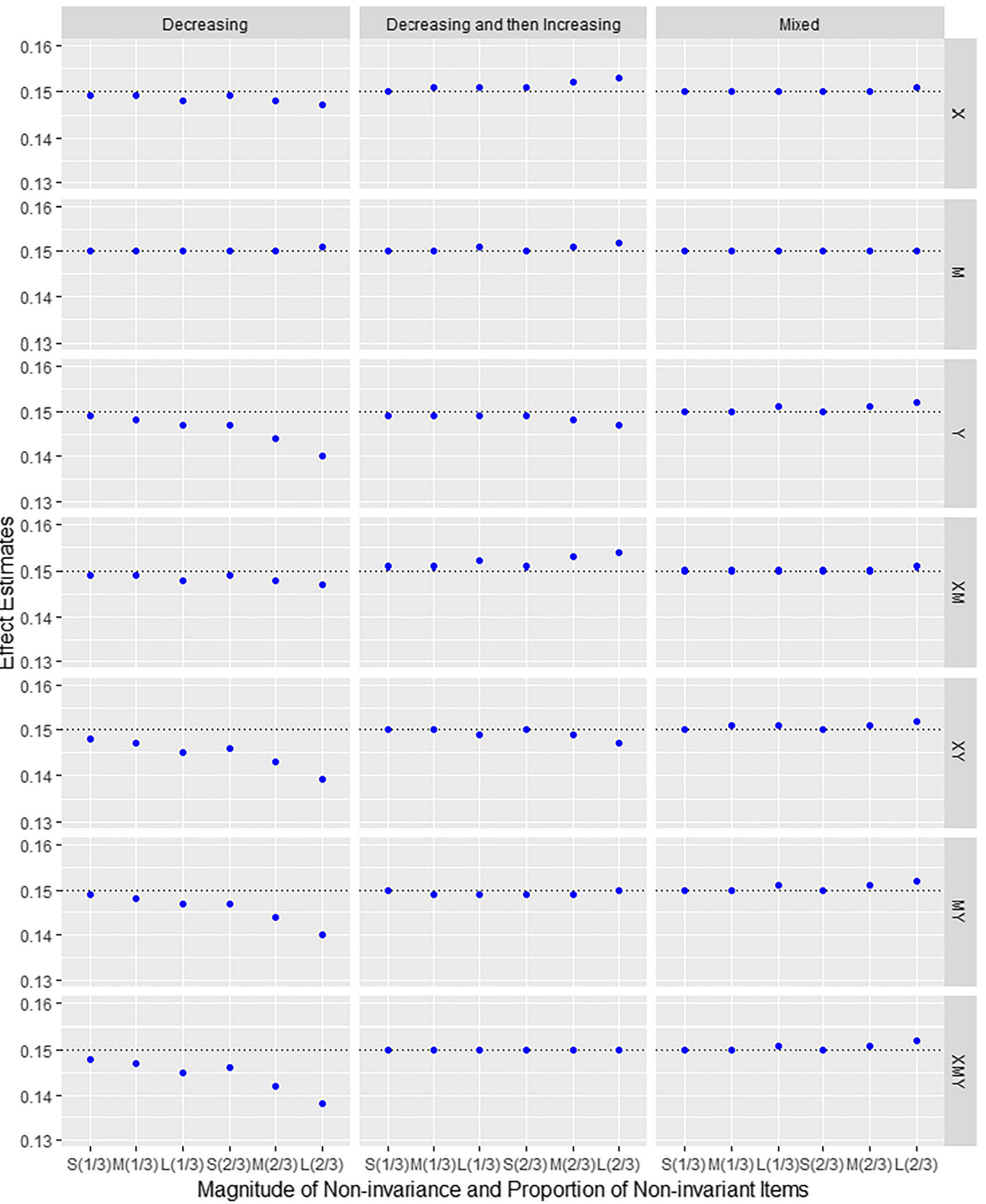

Fig. 9 Direct effect under the non-invariant loading conditions with partial mediation $\left(a=b=.3\right.$ and $\left.c^{\prime}=.15\right) . X, M, Y, X M, X Y, M Y$, and $X M Y$ denote the role of latent variables with non-invariant item parameters

playing in the model; S, M, and L indicate small, medium, and large magnitude of non-invariance, respectively; $1 / 3$ and $2 / 3$ indicate small and large proportion of non-invariant items, respectively 
Table 3 Summary of non-invariance conditions showing relatively large impact on direct and mediated effects from the population investigation

Estimation of the mediated Estimation of the direct effect effect

\begin{tabular}{|c|c|c|c|c|c|c|c|c|}
\hline \multirow{2}{*}{\multicolumn{3}{|c|}{$\begin{array}{l}\text { Decreasing loading } \\
\text { Nonzero mediated effect, } \\
\text { zero or nonzero direct } \\
\text { effect: } a=b=.3, c^{\prime}=0 \\
a=b=.3, c^{\prime}=.15\end{array}$}} & \multicolumn{6}{|c|}{ Decreasing loading } \\
\hline & & & \multicolumn{3}{|c|}{$\begin{array}{l}\text { Non-zero direct and } \\
\text { mediated effects: } \\
a=b=.3, c^{\prime}=.15\end{array}$} & \multicolumn{3}{|c|}{$\begin{array}{l}\text { Zero mediated } \\
\text { effect, nonzero } \\
\text { direct effect: } \\
a=.3, b=0, c^{\prime}= \\
.15 \\
a=0, b=.3, c^{\prime}= \\
.15 \\
a=b=0, c^{\prime}=.15\end{array}$} \\
\hline$M$ & $2 / 3$ & $\mathrm{Lg}$ & $Y$ & $2 / 3$ & $\operatorname{Lg}$ & $Y$ & $2 / 3$ & $\mathrm{Lg}$ \\
\hline$Y$ & $2 / 3$ & $\operatorname{Lg}$ & $X Y$ & $2 / 3$ & $\mathrm{Lg}$ & $X Y$ & $2 / 3$ & $\mathrm{Lg}$ \\
\hline$X M$ & $2 / 3$ & Md or Lg & $M Y$ & $2 / 3$ & $\mathrm{Lg}$ & $M Y$ & $2 / 3$ & $\mathrm{Lg}$ \\
\hline$X Y$ & $2 / 3$ & Md or Lg & $X M Y$ & $2 / 3$ & Md or $\mathrm{Lg}$ & $X M Y$ & $2 / 3$ & $\mathrm{Lg}$ \\
\hline$M Y$ & $2 / 3$ & Md or Lg & & & & & & \\
\hline$X M Y$ & $1 / 3$ & $\mathrm{Lg}$ & & & & & & \\
\hline$X M Y$ & $2 / 3$ & Md or Lg & & & & & & \\
\hline
\end{tabular}

Note: $M, Y, X M, X Y, M Y$, and $X M Y$ indicate role of latent variables with non-invariant items; $1 / 3$ and $2 / 3$ is the proportion of non-invariant items; $\mathrm{Md}$ and $\mathrm{Lg}$ indicate the magnitude of non-invariance: Md means medium non-invariance (a change of 0.2 ) and $\mathrm{Lg}$ means large non-invariance (a change of 0.3 )

\section{Investigation based on sample covariance matrices}

To examine statistical inferences regarding the direct and mediated effects and to examine whether model fit indices can help detect longitudinal measurement non-invariance under finite samples, we generated sample data under conditions with non-invariant items in $M$ and $Y$, or all of $X, M$, and $Y$. We also included conditions under longitudinal measurement invariance. Both loadings and item residual variances were constrained as invariant across time under the invariant conditions. Furthermore, the following conditions were considered for direct and indirect effects: (1) $a=b=.3$ and $c^{\prime}=0$, (2) $a=b=.3$ and $c^{\prime}=.15$, and (3) $a=.3, b=0$, and $c^{\prime}=.15$. Conditions (1) and (2) had non-zero mediated effect, and conditions (2) and (3) had non-zero direct effect. The three combinations of $a, b$, and $c^{\prime}$ were used for both measurement invariance and non-invariance scenarios.

Three levels of sample size $(200,500$, and 1000) were selected for data generation. Therefore, we had nine invariance conditions. When longitudinal measurement invariance was violated, we only considered metric non-invariance. We selected conditions with $2 / 3$ items decreasing their loadings by a maximum difference of .3 (large) for non-invariant variables. These conditions in combination with our selected effects of $a, b$, and $c^{\prime}$ were used because they produced the least accurate direct and mediated effect estimates from the analyses based on population covariance matrices. In total, we had 18 non-invariance conditions. Five hundred datasets were generated for each condition. The analysis models were the same as those based on population covariance matrices. For each replication, 500 bootstrap samples were used to construct the BC confidence intervals. Mplus7 (Muthén \& Muthén, 1998-2012) was used to estimate models using the ML estimator via the MplusAutomation package (Hallquist \& Wiley, 2013) installed in the R program (R Core Team, 2016).

\section{Overall model fit}

The overall model fit was assessed using the Chi-square test, CFI, and RMSEA. Note that for the measurement invariance conditions, the expected value of the Chi-square statistic was model degrees of freedom, $\mathrm{CFI}=1$, and RMSEA $=0$. For the measurement non-invariance conditions, the expected value of the Chi-square statistic was model degrees of freedom plus $(n-1)$ times fit function that was obtained from the corresponding analysis based on population covariance matrices. Expected values of CFI and RMSEA were obtained from the corresponding analysis based on the population covariance matrix.

The left panel in Table 4 shows Chi-square statistic, CFI, and RMSEA values that were expected for each of the 27 conditions. The right panel in the table shows the mean of Chi-square statistics, the mean of CFI values, and the mean of RMSEA values across all 500 samples, as well as the model rejection rates based on Chi-square test at the alpha level of .05 . Under both invariance and non-invariance conditions, the mean Chi-square statistic from the finite samples was larger than the expected values. This indicates that the Chi-square test did not perform well under finite samples, and the performance was worse under smaller sample sizes. Consequently, the correctly specified model was overly rejected across all sample sizes we considered and the power for detecting longitudinal measurement non-invariance was spuriously high under small sample sizes.

We adopted the conventional criteria, CFI $\geq .95$ and RMSEA $\leq .05$ to indicate good model-data fit ( $\mathrm{Hu} \&$ Bentler, 1999). We found that these fit indices did not lead to the same conclusion as the model Chi-square test, and their values deviated from the expected values especially when the sample size was 200. Specifically, RMSEA suggested good model fit for all 27 conditions. For both invariance and noninvariance conditions, CFI suggested an acceptable model fit and the mean CFI was very close to the expected values when the sample size was at least 500; however, CFI indicated a poor fit and the mean CFI was much smaller than the expected values when the sample size was 200 . Holding constant the 
Table 4 Model-data fit under the 27 conditions based on sample covariance matrices

\begin{tabular}{|c|c|c|c|c|c|c|c|c|}
\hline \multirow[t]{2}{*}{ Sample size } & \multirow[t]{2}{*}{ Role } & \multicolumn{3}{|l|}{ Expected } & \multicolumn{4}{|l|}{ Observed } \\
\hline & & $x^{2}$ & $\mathrm{CFI}$ & RMSEA & $\begin{array}{l}\text { Mean } \\
\chi^{2}\end{array}$ & Rejection rate & Mean CFI & Mean RMSEA \\
\hline \multicolumn{9}{|c|}{$a=b=.3$ and $c^{\prime}=0$} \\
\hline \multirow[t]{3}{*}{200} & -- & 4047 & 1.000 & 0 & 4894.61 & 1.000 & .897 & .032 \\
\hline & MY & 4047.64 & .988 & .010 & 4886.52 & 1.000 & .870 & .034 \\
\hline & XMY & 4082.46 & .981 & .012 & 4925.00 & 1.000 & .852 & .034 \\
\hline \multirow[t]{3}{*}{500} & -- & 4047 & 1.000 & 0 & 4327.40 & .900 & .985 & .012 \\
\hline & MY & 4157.14 & .988 & .010 & 4437.01 & .998 & .971 & .015 \\
\hline & XMY & 4244.46 & .981 & .012 & 4521.96 & 1.000 & .961 & .017 \\
\hline \multirow[t]{3}{*}{1000} & -- & 4047 & 1.000 & 0 & 4179.67 & .410 & .996 & .005 \\
\hline & MY & 4339.64 & .988 & .010 & 4472.55 & 1.000 & .984 & .011 \\
\hline & XMY & 4514.46 & .981 & .012 & 4653.34 & 1.000 & .976 & .013 \\
\hline \multicolumn{9}{|c|}{$a=.3, b=0$, and $c^{\prime}=.15$} \\
\hline \multirow[t]{3}{*}{200} & -- & 4047 & 1.000 & 0 & 4889.48 & 1.000 & .897 & .032 \\
\hline & MY & 4048.03 & .988 & .010 & 4887.54 & 1.000 & .868 & .034 \\
\hline & XMY & 4082.66 & .980 & .012 & 4923.62 & 1.000 & .850 & .034 \\
\hline \multirow[t]{3}{*}{500} & -- & 4047 & 1.000 & 0 & 4329.47 & .918 & .985 & .012 \\
\hline & MY & 4158.13 & .988 & .010 & 4428.91 & 1.000 & .971 & .015 \\
\hline & XMY & 4244.96 & .980 & .012 & 4529.71 & 1.000 & .960 & .017 \\
\hline \multirow[t]{3}{*}{1000} & -- & 4047 & 1.000 & 0 & 4174.35 & .386 & .996 & .005 \\
\hline & MY & 4341.63 & .988 & .010 & 4475.73 & 1.000 & .984 & .011 \\
\hline & XMY & 4515.46 & .980 & .012 & 4647.10 & 1.000 & .976 & .013 \\
\hline \multicolumn{9}{|c|}{$a=b=.3$, and $c^{\prime}=.15$} \\
\hline \multirow[t]{3}{*}{200} & -- & 4047 & 1.000 & 0 & 4884.05 & 1.000 & .900 & .032 \\
\hline & MY & 4047.83 & .988 & .010 & 4885.78 & 1.000 & .873 & .034 \\
\hline & XMY & 4082.46 & .981 & .012 & 4929.37 & 1.000 & .854 & .035 \\
\hline \multirow[t]{3}{*}{500} & -- & 4047 & 1.000 & 0 & 4322.77 & .912 & .986 & .011 \\
\hline & MY & 4157.63 & .988 & .010 & 4440.93 & 1.000 & .971 & .015 \\
\hline & XMY & 4244.46 & .981 & .012 & 4525.46 & 1.000 & .962 & .017 \\
\hline \multirow[t]{3}{*}{1000} & -- & 4047 & 1.000 & 0 & 4181.49 & .420 & .996 & .005 \\
\hline & MY & 4340.63 & .988 & .010 & 4471.13 & 1.000 & .984 & .011 \\
\hline & XMY & 4514.46 & .981 & .012 & 4652.89 & 1.000 & .976 & .013 \\
\hline
\end{tabular}

Note: role means the role of latent variable with non-invariant items playing in the model; - means measurement invariance conditions. Degrees of freedom were 3975 and 4047 for the non-invariant and invariant conditions, respectively

sample size, model fit for conditions with non-invariant items in $X M Y$ was slightly worse than conditions with non-invariant items in $M Y$ because of the increase in the degree of model misspecification. The impact of the size of structural paths ( $a$, $b$, and $c^{\prime}$ ) was minimal on the Chi-square statistic and the investigated fit indices.

\section{Parameter estimation and significance testing}

Under each condition, relative bias of parameter estimate was computed as:
$\operatorname{RB}(\widehat{\theta})=\sum_{l=1}^{L}\left(\frac{\widehat{\theta}_{l}-\theta}{\theta}\right) / L$

where $\widehat{\theta}_{l}$ is the estimated parameter of interest (i.e., the direct and mediated effects) from the $t^{\text {th }}$ replication, $\theta$ is the true parameter, and $L$ is the converged number of replications. Magnitude of relative bias greater than .05 was considered large in terms of Hoogland and Boomsma's (1998) criterion. Coverage rate was calculated as the proportion of the estimated 95\% BC bootstrap CIs containing the true population effects. For a 95\% confidence interval, the acceptable coverage 
rate ranges from .925 to .975 in terms of Bradley's liberal criterion (1978).

For all 27 conditions, all bootstrap samples for each replication converged to proper solutions. Table 5 provides bias/ relative bias and coverage rate for parameters of interest under the 27 conditions. The values were bold if the size of relative bias was greater than .05 or coverage rate was outside the acceptable range. Across all invariance conditions, bias/ relative bias for all parameter estimates $(a, b$, the mediated effect, and the direct effect) were negligible. Also, coverage rates for the direct and mediated effects were in general within the acceptable range.

When the true effects were non-zero under the noninvariance conditions, relative bias of the direct and mediated effect estimates was larger than .05 in magnitude. When the direct or mediated effect was zero in the data generating model, bias of effect estimates was minimal. Coverage rates for the mediated effect were below .90 across all non-invariance conditions when the mediated effect was non-zero, and were above .90 when the mediated effect was zero. Coverage rates for the direct effect were generally within the acceptable range when either the direct or the mediated effect was zero in the population, and can be lower than .90 when both the direct and mediated effect were non-zero. Note that relatively low coverage rates indicate relatively high type I error rates with zero effects in the population. Power for detecting the direct and mediated effects was equal to or very close to 1.0 across all non-invariant conditions.

It can be noted that, when the true effects were non-zero, as sample size increased, coverage rate for these effects tended to be lower across non-invariance conditions. In contrast, under invariance conditions (Table 5), coverage rate was not sensitive to sample size. This is because estimates of the non-zero true effects were generally biased under the non-invariance conditions and the $\mathrm{BC}$ confidence intervals tended to be narrower for larger sample sizes and thus were less likely to capture the true effect. In contrast, parameter estimates were generally accurate under the invariance conditions, and thus the $\mathrm{BC}$ confidence intervals can capture the true effect regardless of the sample size.

Table 5 Bias/relative bias and coverage rate for parameter estimates under the non-invariant loading conditions

\begin{tabular}{|c|c|c|c|c|c|c|c|}
\hline \multirow[t]{2}{*}{ Sample size } & \multirow[t]{2}{*}{ Role } & \multirow{2}{*}{$\begin{array}{l}a \text { path } \\
R B\end{array}$} & \multirow{2}{*}{$\begin{array}{l}b \text { path } \\
R B / \text { bias }\end{array}$} & \multicolumn{2}{|c|}{ Mediated effect $(a * b)$} & \multicolumn{2}{|c|}{ Direct effect $\left(c^{\prime}\right)$} \\
\hline & & & & $R B /$ bias & Coverage rate & $R B /$ bias & Coverage rate \\
\hline \multicolumn{8}{|c|}{$a=b=.3$ and $c^{\prime}=0$} \\
\hline \multirow[t]{3}{*}{200} & -- & .006 & -.005 & -.001 & .944 & .002 & .934 \\
\hline & MY & -.056 & -.066 & -.119 & .892 & -.002 & .938 \\
\hline & XMY & -.057 & -.063 & -.118 & .890 & .000 & .946 \\
\hline \multirow[t]{3}{*}{500} & -- & .005 & .004 & .009 & .934 & .000 & .952 \\
\hline & MY & -.066 & -.059 & -.121 & .774 & .000 & .946 \\
\hline & XMY & -.072 & -.060 & -.128 & .758 & -.001 & .938 \\
\hline \multirow{3}{*}{1000} & -- & .003 & .004 & .007 & .952 & -.001 & .944 \\
\hline & MY & -.063 & -.061 & -.121 & .620 & -.001 & .936 \\
\hline & XMY & -.072 & -.062 & -.130 & .572 & -.001 & .950 \\
\hline \multicolumn{8}{|c|}{$a=.3, b=0$, and $c^{\prime}=.15$} \\
\hline \multirow[t]{3}{*}{200} & -- & .002 & .000 & .000 & .936 & -.017 & .954 \\
\hline & MY & -.064 & .000 & .000 & .914 & -.067 & .940 \\
\hline & XMY & -.059 & -.001 & .000 & .918 & -.062 & .948 \\
\hline \multirow[t]{3}{*}{500} & -- & .000 & .000 & .000 & .948 & .001 & .958 \\
\hline & MY & -.062 & .000 & .000 & .928 & -.056 & .952 \\
\hline & XMY & -.071 & -.001 & .000 & .926 & -.071 & .930 \\
\hline \multirow[t]{3}{*}{1000} & -- & -.002 & .000 & .000 & .942 & .003 & .936 \\
\hline & MY & -.058 & .001 & .000 & .920 & -.057 & .908 \\
\hline & XMY & -.071 & .001 & .000 & .934 & -.066 & .906 \\
\hline \multicolumn{8}{|c|}{$a=b=.3$ and $c^{\prime}=.15$} \\
\hline \multirow[t]{3}{*}{200} & -- & .001 & -.002 & -.002 & .944 & -.004 & .948 \\
\hline & MY & -.068 & -.062 & -.127 & .882 & -.059 & .942 \\
\hline & XMY & -.067 & -.059 & -.126 & .886 & -.077 & .932 \\
\hline \multirow[t]{3}{*}{500} & -- & .002 & -.001 & .000 & .954 & .001 & .966 \\
\hline & MY & -.067 & -.062 & -.126 & .756 & -.057 & .896 \\
\hline & XMY & -.071 & -.064 & -.131 & .750 & -.082 & .908 \\
\hline \multirow{3}{*}{1000} & -- & -.001 & .000 & -.002 & .936 & .007 & .934 \\
\hline & MY & -.063 & -.062 & -.121 & .608 & -.066 & .882 \\
\hline & XMY & -.069 & -.062 & -.127 & .594 & -.083 & .838 \\
\hline
\end{tabular}

Note: $R B$ means relative bias. Role means the role of latent variable with non-invariant items playing in the model; - means measurement invariance conditions. Values were bold if $R B$ for parameter estimates was greater than .05 in magnitude or coverage rate was below the lower bound of the acceptable range (.925) 


\section{Discussion and conclusions}

In this study, we investigated influence of longitudinal measurement non-invariance on parameter estimates and model fit under the latent cross-lagged panel mediation model (LCLPM) via a simulation study. Specifically, analyses were conducted using population covariance matrices and finite samples. Analyses based on population covariance matrices showed that non-invariance in item residual variances had minimal effect on parameter estimates, whereas that in loadings had meaningful impact. Therefore, in finite sample analyses, we considered violation of metric invariance only. Combining results from both the population and sample investigation, we have several findings.

First, as the magnitude of non-invariance, number of noninvariant items, and/or the proportion of non-invariant items increased, direct and mediated effect estimates tended to be less accurate. These conclusions were consistent with studies investigating the impact of measurement non-invariance on parameter estimation accuracy in the literature (e.g., Liang et al., 2018; Olivera-Aguilar, 2013). Second, three patterns of non-invariant loadings were examined with roughly the same variability of loadings over time: monotonically decreasing or increasing, increasing and then decreasing, and mixed (half of non-invariant items had increasing loadings and the other had decreasing loadings). Results indicated that the monotonically decreasing or increasing pattern resulted in the most inaccurate estimates of the direct and mediated effects. The direct and mediated effect estimates were generally accurate (relative bias $<5 \%$ ) when item parameters changed over time following the decreasing and then increasing pattern or the mixed pattern. For the decreasing and then increasing pattern, $a$ and $b$ were not simultaneously over- or under-estimated. Instead, one can be over-estimated, and the other was under-estimated, resulting in relatively accurate estimates of $a b$. For the mixed pattern, effects of simultaneous decreasing and increasing changes in different items may be canceled out in the estimation of $a, b$, and $c^{\prime}$, resulting in reasonably accurate estimates of the direct and mediated effects. Third, with non-invariant loadings, type I error rates for testing the mediated effects based on the $\mathrm{BC}$ bootstrap $\mathrm{CI}$ can be inflated (e.g., higher than .075) with relatively small sample size (e.g., 200). Mediated effects and direct effects tended to be under-covered (coverage rates $<.9$ ) when they were non-zero in the population.

Previous research showed that the BC bootstrap method can yield inflated type I error rates for the mediated effect in cross-sectional mediation models under conditions where the population mediated effect was zero (i.e., $a$ path was zero or $b$ path was zero; Cheung \& Lau, 2008; Fritz, Taylor, \& MacKinnon, 2012; Hayes \& Scharkow, 2013; Valente et al., 2016). However, results from the current study showed that type I error rate was generally acceptable under zero effect condition with relatively large sample sizes. Therefore, conclusions about type I error rate of the mediated effect based on the $\mathrm{BC}$ bootstrap $\mathrm{CI}$ from previous research were not held for longitudinal mediated effect.

Second, fit indices were not sensitive to misspecifications caused by measurement non-invariance in the investigated LCLPMs based on the commonly used evaluation criteria. For the 18 conditions that yielded the most inaccurate direct and indirect effect estimates in the population covariance matrix analyses, the CFI values were all greater than .98 and RMSEA values were all smaller than .012. Marsh, Hau, and Wen (2004) argued that such models should be considered as "acceptable-misspecified models". Our finite-sample analyses further revealed that the fit indices deviated from the expected values when the sample size was 200 . This result suggests that fit indices tend to yield misleading decision regarding modeldata fit in L-CLPMs when the sample size is small. The unsatisfactory performance of fit indices in L-CLPM is likely due to model complexity. As such, we do not recommend the application of L-CLPM unless the sample size is sufficiently large.

Third, in finite-sample analyses, the type I error rate for the Chi-square test of model fit was seriously inflated, especially when the sample size was relatively small. This is consistent with the existing finding that the Chi-square statistic can reject the correctly specified model when the model is complex (e.g., our studied L-CLPM) and/or the sample size is relatively small (Bearden, Sharma, \& Teel, 1982; Hoogland \& Boomsma, 1998; Zhang \& Yang, in press). Specifically, the sampling distribution of the Chi-square statistics deviates from the central Chi-square distribution with relatively small sample sizes and/or large model size.

\section{Extension to the scenario with correlated measurement errors}

In our simulation study, measurement errors were assumed to be uncorrelated in both population and sample level investigations. In practice, often times measurement errors of the same item are correlated over time (e.g., Cole \& Maxwell, 2003). Therefore, a small-scale simulation was conducted at the sample level when measurement errors of the same item were correlated at adjacent time points (with a correlation of .3). We studied under the same conditions as our sample investigation with non-invariant items in $M Y$, or $X M Y, 2 / 3$ items decreasing their loadings by a maximum difference of .3 (large), and three combinations of $a, b$, and $c^{\prime}$ values. A sample size of 200 was used. All datasets were fitted to L-CLPMs with correlated measurement errors at the adjacent time points. As expected, with the correlated measurement errors being correctly specified in the fitted models, results regarding parameter estimates and model fit were similar to the scenarios with uncorrelated measurement errors in the population. 


\section{Suggestions and implications}

When using L-CLPMs, we have several suggestions. First, it is important to examine the longitudinal measurement invariance assumption rather than assuming it is met. Estimates of the direct and mediated effects with violations of longitudinal invariance can be considerably biased when loadings are varied over time. Second, it is important to check the pattern of non-invariance over time in the applied model. Pattern of longitudinal noninvariance can be observed by freely estimating all item parameters (e.g., loadings and item residual variances) except for those that are constrained for model identification purpose. As is shown in our study, caution should be needed when the loadings change in one direction (either decreasing or increasing) for a large number of items, particularly in the mediator and/or outcome. When non-invariance items are detected, researchers may fit the data to a partially invariant model given that the model is identified. In this circumstance, the accuracy of direct and indirect effects would depend on the item used to identify the latent factors. In the current study, the last item was invariant under all considered conditions and was used as the reference indicator in the analysis models. However, in applications invariant items are often unknown a priori. Therefore, detecting non-invariant items and choosing an appropriate reference indicator is crucial before examining direct and indirect effects. On the other hand, if upon a thorough qualitative evaluation of items based on substantive theories and expert review, the researcher decides to include noninvariant items in the scale for construct representation purpose (Shi, Song, \& Lewis, 2017), then a modeling strategy should be adopted to examine the type and pattern of non-invariance. Equality constraints should not be placed on the loadings of items if loadings change monotonically, especially with large magnitude of non-invariance.

Third, conclusions about model fit based on the Chi-square test and model fit indices with the commonly used evaluation criteria can be misleading under L-CLPM especially under small samples. Echoing Zhang and Yang (in press), Chisquare test of model fit and model fit indexes tend to be more trustworthy when sample size is relatively large (e.g., >1000) and/or the L-CLPM is relatively simple (e.g., with a small number of indicators per latent factor).

\section{Limitations and future directions}

The study has several limitations that may enlighten future research. First, to cope with the problems of model-data fit test and indices, it is worthwhile to consider potential alternatives for detecting measurement non-invariance in crosslagged panel mediation models. For example, instead of comparing fit indices to the established criteria on absolute fit, one might establish criteria of meaningful differences in model fit indices between nested models when evaluating longitudinal measurement invariance.
Second, this study considers continuous data following multivariate normal distributions. In practice, continuous data often deviate from normality and ordinal data have been commonly used. Future investigations may focus on the impact of longitudinal measurement non-invariance on mediation analysis in the L-CLPM with non-normal or categorical indicators.

Third, the current study focuses on the impact of measurement non-invariance on structural parameter estimates and model fit in L-CLPMs. The magnitude of mediated effects is dependent on the time lag (Cole \& Maxwell, 2003). It should be noted that the selection of time intervals between adjacent measurements may also affect parameter estimation and model fit, since the direct and mediated effect estimates are "timespecific" (Gollob \& Reichardt, 1991, p. 253) in the current study. To handle limitations with time-specific effects in autoregressive mediation modeling (Gollob \& Reichardt, 1991; Wang \& Zhang, in press), one potential solution is to estimate the "total" direct and mediated effect for a certain time lag in addition to the time-specific effects (Little, 2013; Wang \& Zhang, in press). Wang and Zhang (in press) showed that the total effects are asymptotically invariant with different time intervals. Future studies may consider this approach to address the issue of time-specific effect estimates in crosslagged panel mediation modeling.

Last, in the context of multiple-group analysis, Shi et al. (2017) showed that under partial invariance, selecting an incorrect reference indicator can produce noticeable estimation bias under the non-invariant factor loading conditions. Xu and Green (2016) examined how the number of invariance constraints impacted model fit and estimation of latent mean difference. In the current study, partially invariant models allowing time-varying parameter estimates for some items were not considered as analysis models. We expect that given the correct choice of reference indicator, results from partially invariant models would be very close to the population values when fitting the partially invariant models to data because the fitted models are consistent with the data generation models. We also expect that if a non-invariant item is mistakenly chosen as the reference indicator, the estimated direct and indirect effects would be inaccurate. However, further studies may be conducted to examine whether and how a wrong choice of reference indicator and incorrect detection of non-invariant parameters interact with each other and impact the estimated direct and mediated effects in LCPM models.

\section{References}

Bearden, W. O., Sharma, S., \& Teel, J.E. (1982). Sample size effects on chi square and other statistics used in evaluating causal models. Journal of Marketing Research, XIX, 425-30. 
Bentley, J. P. (2011). An examination of statistical methods for longitudinal mediation modeling (Doctoral dissertation, University of Alabama at Birmingham). Retrieved from http://www.mhsl.uab. edu/dt/2011p/bentley.pdf.

Boom, J. (2015). A new visualization and conceptualization of categorical longitudinal development: Measurement invariance and change. Frontiers in Psychology, 6(289), 32-48.

Bradley, J. V. (1978). Robustness? British Journal of Mathematical and Statistical Psychology, 31(2), 144-152.

Cheong, J., MacKinnon, D. P., \& Khoo, S. T. (2003). Investigation of meditational processes using parallel process latent growth curve modeling. Structural Equation Modeling, 10, 238-262.

Cheung, G. W., \& Lau, R. S. (2008). Testing mediation and suppression effects of latent variables: Bootstrapping with structural equation models. Organizational Research Methods, 11(2), 296-325.

Cohen, J. (1988). Statistical power analysis for the behavioral sciences (2nd). Hillsdale, NJ: Lawrence Erlbaum Associates.

Cole, D. A., \& Maxwell, S. E. (2003). Testing mediation models with longitudinal data: Questions and tips in the use of structural equation modeling. Journal of Abnormal Psychology, 112, 558-577.

Fritz, M. S., Taylor, A. B., \& MacKinnon, D. P. (2012). Explanation of two anomalous results in statistical mediation analysis. Multivariate Behavioral Research, 47, 61-87.

Gollob, H. F., \& Reichardt, C. S. (1987). Taking account of time lags in causal models [Special section on structural equation modeling]. Child Development, 58, 80-92.

Gollob, H. F., \& Reichardt, C. S. (1991). Interpreting and estimating indirect effects assuming time lags really matter. In Collins, L. M., Horn, J. L. (Eds.). In Best methods for the analysis of change (p. 243-259). Washington, DC: American Psychological Association.

Gonzalez, O., Valente, M. J., \& MacKinnon, D. P. (2017). Longitudinal measurement invariance in a two-wave mediation model. Paper presented at the annual meeting of the Society for Prevention Research, Washington, DC

Guenole, N., \& Brown, A. (2014). The consequences of ignoring measurement invariance for path coefficients in structural equation models. Frontiers in Psychology, 5, 1-16.

Hallquist, M., \& Wiley, J. (2013). MplusAutomation: Automating Mplus model estimation and interpretation ( $\mathrm{R}$ package version $0.5-4)$. Retrieved from https://CRAN.R-project.org/package $=$ MplusAutomation.

Hanson, LLM, Peristera, P., Chungkham, H.S., \& Westerlund, H. (2016). Longitudinal mediation modeling of unhealthy behaviors as mediators between workplace demands/support and depressive symptoms. PLOS ONE, 11(12): e 0169276.

Hayes, A. F., \& Scharkow, M. (2013). The relative trustworthiness of inferential tests of the indirect effect in statistical mediation analysis: Does method really matter? Psychological Science, 24, 1918-1927.

Hoogland, J. J., \& Boomsma, A. (1998). Robustness studies in covariance structure modeling: An overview and a meta-analysis. Sociological Methods \& Research, 26, 329-367.

Hu, L.-T., \& Bentler, P. M. (1999). Cutoff criteria for fit indexes in covariance structure analysis: Conventional criteria versus new alternatives. Structural Equation Modeling, 6, 1-55.

Jaya, E. S., Ascone, L., \& Lincoln, T. M. (2018). A longitudinal mediation analysis of the effect of negative-self-schemas on positive symptoms via negative affect. Psychological Medicine, 48(8), 1299-1307.

Judd, C. M., \& Kenny, D. A. (1981). Process analysis: Estimating mediation in treatment evaluations. Evaluation Review, 5, 602-619.

Kenny, D. A. (1979). Correlation and causality. New York: Wiley.

Leite, W. L. (2007). A comparison of latent growth models for constructs measured by multiple items. Structural Equation Modeling, 14, 581-610.

Liang, X., Yang, Y., \& Huang, J. (2018). Evaluation of structural relationships in autoregressive cross-lagged models under longitudinal approximate invariance: A Bayesian analysis. Structural Equation Modeling. 25(4), 558-572.

Little, T. D. (2013). Longitudinal structural equation modeling. New York, NY: Guilford.

Liu, Y., Millsap, R. E., West, S. G., Tein, J.-Y., Tanaka, R., \& Grimm, K. J. (2016). Testing measurement invariance in longitudinal data with ordered-categorical measures. Psychological Methods, 22(3), 486506.

MacKinnon, D. P. (2008). Introduction to Statistical Mediation Analysis. New York: Erlbaum.

Marsh, H. W., Hau, Kit-T., \& Wen, Z. (2004). In Search of Golden Rules: Comment on Hypothesis-Testing Approaches to Setting Cutoff Values for Fit Indexes and Dangers in Overgeneralizing $\mathrm{Hu}$ and Bentler's (1999) Findings, Structural Equation Modeling: A Multidisciplinary Journal, 11(3), 320-341.

Maxwell, S. E., \& Cole, D. A. (2007). Bias in cross-sectional analyses of longitudinal mediation. Psychological Methods, 12, 23-44.

Maxwell, S. E., Cole, D. A., \& Mitchell, M. A. (2011). Bias in crosssectional analyses of longitudinal mediation: partial and complete mediation under an autoregressive model. Multivariate Behavioral Research, 46(8), 16-41.

McArdle, J. J. (2009). Latent variable modeling of differences and changes with longitudinal data. Annual Review of Psychology, 60, $577-$ 605.

Mellenbergh, G. J. (1989). Item bias and item response theory. International Journal of Educational Research, 13, 127-143.

Meredith, W. (1993). Measurement invariance, factor analysis and factorial invariance. Psychometrika, 58, 525-543.

Meredith, W., \& Horn, J. (2001). The role of factorial invariance in modeling growth and change. In L. M. Collins \& A.G. Sayer (Eds.). New methods for the analysis of change (pp. 203-240). Washington DC: American Psychological Association.

Meredith, W., \& Millsap, R. E. (1992). On the misuse of manifest variables in the detection of measurement bias. Psychometrika, 57, 289311.

Millsap. R. E. \& Cham, H. (2012). Investigating factorial invariance in longitudinal data. In B. Laursen, T. D., Little, \& N. A. Card. (Ed.), Handbook of Developmental Research Methods (pp. 109-126). NY: Guilford.

Muthén, L. K. \& Muthén, B. O. (1998-2012). Mplus user's guide (7th). Los Angeles, CA: Muthén \& Muthén.

Obradovic, J., Pardini, D. A., Long, J. D., \& Loeber, R. (2007). Measuring interpersonal callousness in boys from adolescence: An examination of longitudinal invariance and temporal stability. Journal of Clinical Child and Adolescent Psychology, 36, 276-292.

Olivera-Aguilar, M. (2013). Impact of violations of longitudinal measurement invariance in latent growth models and autoregressive quasisimplex models (Doctoral dissertation, Arizona State University). Retrieved from https://repository.asu.edu/attachments/114425/ content/OliveraAguilar_asu_0010E_13164.pdf.

Pardini, D. A., Loeber, R., \& Stouthamer-Loeber, M. (2005). Developmental shifts in parent and peer influences on boys' beliefs about delinquent behavior. Journal of Research on Adolescence, 15 , 299-323.

R Core Team. (2016). R: A language and environment for statistical computing. Vienna, Austria: R Foundation for Statistical Computing.

Sass, D. A. (2011). Testing measurement invariance and comparing latent factor means within a confirmatory factor analysis framework. Journal of Psychoeducational Assessment, 29(4), 347-363.

Schlueter, E., Davidov, E., \& Schmidt, P. (2007). Applying autoregressive cross-lagged and latent growth curve models to a three-wave panel study. In K. Van Montfort, J. Oud, \& A. Satorra (Eds.), Longitudinal models in the behavioral and related sciences (pp. 315-336). Mahwah, NJ: Lawrence Erlbaum Associates. 
Selig, J. P., \& Little, T. D. (2012). Autoregressive and cross-lagged panel analysis for longitudinal data. In B. Laursen, T. D. Little, \& N. A. Card (Eds.), Handbook of developmental research methods (pp. 265-278). New York: Guilford Press.

Selig, J. P., \& Preacher, K. J. (2009). Mediation models for longitudinal data in developmental research. Research in Human Development, $6,144-164$.

Shi, D., Song, H., \& Lewis, M. D. (2017). The impact of partial factorial invariance on cross-group comparisons. Assessment, 1-17.

Soest, T. V., \& Hagtvet, K. A. (2011). Mediation analysis in a latent growth curve modeling framework. Structural Equation Modeling: A Multidisciplinary Journal, 18(2), 289-314.

Valente, M. J., Gonzalez, O., Miočević, M., \& MacKinnon, D. P. (2016). A note on testing mediated effects in structural equation models: Reconciling past and current research on the performance of the test of joint significance. Educational and Psychological Measurement, 76, 889-911.

Valeri, L., \& VanderWeele, T. J. (2013). Mediation analysis allowing for exposure-mediator interactions and causal interpretation: Theoretical assumptions and implementation with SAS and SPSS macros. Psychological Methods, 18(2), 137-150.

Wang, L., \& Zhang, Q. (in press). Investigating the impact of the time interval selection on autoregressive mediation modeling: Result interpretations, effect reporting, and temporal designs. Psychological Methods.

Watts, P., Webb, E., \& Netuveli, G. (2017). The role of sports clubs in helping older people to stay active and prevent frailty: A longitudinal mediation analysis. International Journal of Behavioral Nutrition and Physical Activity, 14 (95), 1-9.

Widaman, K. F., Ferrer, E., \& Conger, R. D. (2010). Factorial invariance within longitudinal structural equation models: Measuring the same construct across time. Child Development Perspectives, 4(1), 10-18.

Willoughby, M. T., Wirth, R. J., \& Blair, C. B. (2012). Executive function in early childhood: Longitudinal measurement invariance and developmental change. Psychological Assessment, 24, 418-431.

Xu, Y. \& Green, S. B. (2016). The impact of varying the number of measurement invariance constraints on the assessment of betweengroup differences of latent means. Structural Equation Modeling: A Multidisciplinary Journal, 23(2), 290-301.

Zhang, Q., \& Yang, Y. (in press). Autoregressive mediation models using composite scores and latent variables: Comparisons and recommendations. Psychological Methods.

Publisher's note Springer Nature remains neutral with regard to jurisdictional claims in published maps and institutional affiliations. 